\title{
A revelation principle for obviously strategy-proof implementation
}

Citation for published version (APA):

Mackenzie, A. (2018). A revelation principle for obviously strategy-proof implementation. Maastricht University, Graduate School of Business and Economics. GSBE Research Memoranda No. 014 https://doi.org/10.26481/umagsb.2018014

Document status and date:

Published: 08/05/2018

DOI:

10.26481/umagsb.2018014

Document Version:

Publisher's PDF, also known as Version of record

\section{Please check the document version of this publication:}

- A submitted manuscript is the version of the article upon submission and before peer-review. There can be important differences between the submitted version and the official published version of record.

People interested in the research are advised to contact the author for the final version of the publication, or visit the DOI to the publisher's website.

- The final author version and the galley proof are versions of the publication after peer review.

- The final published version features the final layout of the paper including the volume, issue and page numbers.

Link to publication

\footnotetext{
General rights rights.

- You may freely distribute the URL identifying the publication in the public portal. please follow below link for the End User Agreement:

www.umlib.nl/taverne-license

Take down policy

If you believe that this document breaches copyright please contact us at:

repository@maastrichtuniversity.nl

providing details and we will investigate your claim.
}

Copyright and moral rights for the publications made accessible in the public portal are retained by the authors and/or other copyright owners and it is a condition of accessing publications that users recognise and abide by the legal requirements associated with these

- Users may download and print one copy of any publication from the public portal for the purpose of private study or research.

- You may not further distribute the material or use it for any profit-making activity or commercial gain

If the publication is distributed under the terms of Article $25 \mathrm{fa}$ of the Dutch Copyright Act, indicated by the "Taverne" license above, 


\section{Maastricht University}

Andrew Mackenzie

A revelation principle for obviously strategy-proof implementation

$\mathrm{RM} / 18 / 014$

\section{GSBE}

Maastricht University School of Business and Economics

Graduate School of Business and Economics

P.O Box 616

NL- 6200 MD Maastricht

The Netherlands 


\title{
A REVELATION PRINCIPLE FOR OBVIOUSLY STRATEGY-PROOF IMPLEMENTATION
}

\author{
ANDREW MACKENZIE* \\ This draft: April 9, 2018
}

\begin{abstract}
We prove that if a stochastic (social choice) rule has an obviously strategy-proof (OSP) implementation ( $\mathrm{Li}, 2016)$, then it has such an implementation through a randomized round table mechanism, where the administrator randomly selects a game form in which the agents take turns making public announcements about their private information. When restricted to deterministic rules, our result improves upon other recent revelation principles by relaxing all recall requirements and by allowing all game trees compatible with normal forms (Alós-Ferrer and Ritzberger, 2016); we also establish robustness to player randomization using novel solution concepts involving mixed strategies and behavioral strategies. We use our result to provide a justification for ordinal mechanisms in the spirit of Carroll (2017), and we provide a simple characterization of the deterministic rules with OSP-implementations using deterministic round table mechanisms and ordinary strategy-proofness.
\end{abstract}

Keywords: obvious strategy-proofness, revelation principle, randomized round table mechanism

\section{Introduction}

\subsection{Overview}

A group of agents who wish to condition a decision on their collective information according to some rule may face a problem familiar in politics and economics: this collective information is known to nobody in its entirety. Such a situation can merit the design of an institution called a game form (or mechanism), which specifies an unambiguous procedure by which the agents make choices to ultimately determine their fate. The plausibility of such an institution succeeding is precisely the plausibility of the agents using what they individually know to make choices that lead to, or implement, the desired outcome, and this is typically articulated using a solution concept: a list of plausible equilibria for every game. In response to experimental evidence challenging the universal

*Department of Economics, Maastricht University, Maastricht, the Netherlands. Email: a.mackenzie@maastrichtuniversity.nl. I thank Gabriel Carroll, Yannai Gonczarowski, Hervé Moulin, Marek Pycia, Yangwei Song, and William Thomson for their comments. I am particularly grateful to Shengwu Li for his thorough feedback and suggestions. 
plausibility of dominant strategy equilibrium, the more demanding notion of obviously dominant strategy equilibrium was recently proposed (Li, 2016), and the objective of this article is to establish a revelation principle promising that any investigation of implementation with this new solution concept - what is called obviously strategy-proof (OSP) implementation - can be safely restricted to a class of canonical game forms.

To understand the new demands of OSP-implementation, recall that one strategy dominates another if it yields an outcome that is at least as desirable no matter what happens. If there are many things that might happen, then, calculating that one strategy dominates another is a rather taxing exercise. On the other hand, if one compares the two strategies only when confronted with the choice of continuing with one or the other, and at this point every outcome the first strategy might yield is at least as desirable as every outcome the second might, then a thorough case-by-case calculation is unnecessary; the first strategy obviously dominates the second. In this way, a strategy might be dominant but not obviously so, and an equilibrium might consist of strategies that are dominant but not obviously so.

Due to these new demands, game forms that were adequate for dominant strategy implementation may fall short for OSP-implementation. Indeed, though the classic revelation principle promises that any investigation of implementation in dominant strategies can be safely restricted to direct mechanisms, where agents simultaneously report what they know (Gibbard, 1973; Myerson, 1981), this is not true for OSP-implementation (Li, 2016). This point is illustrated by a variety of examples:

- for the allocation of one object and money, the second-price auction (Vickrey, 1961) can be OSP-implemented (through an ascending clock auction), but not through its associated direct mechanism (Li, 2016);

- for object allocation, any dual dictatorship rule ${ }^{1}$ can be OSP-implemented, but not through its associated direct mechanism (Troyan, 2016);

- for random object allocation, randomized serial dictatorship can be "random OSPimplemented," but not through its associated direct mechanism (Pycia and Troyan, 2017);

- for the marriage problem, if preferences are "very homogeneous" for one side of the market, then deferred acceptance (Gale and Shapley, 1962) can be OSP-implemented, but not through its associated direct mechanism (Ashlagi and Gonczarowski, 2016); and

- for social choice among ordered alternatives when preferences are single-peaked, any dictarship with safeguards against extremism rule has an OSP-implementation, but not through its associated direct mechanism (Bade and Gonczarowski, 2017; see also Arribillaga, Massó, and Neme, 2017).

Thus an analyst may like to know: is there some canonical class of game forms to which a mechanism designer interested in OSP-implementation can safely restrict attention? The purpose of this paper is to provide the strongest possible reassurance that this is indeed the case, and to do so at a level of generality that covers the above applications and many others.

\footnotetext{
${ }^{1}$ In a dual dictatorship rule, property rights are defined using a priority structure in a narrow class, then Gale's top trading cycles (described in Shapley and Scarf, 1974) is applied.
} 
The usefulness of such a revelation principle to the analysis of OSP-implementation has in fact already been demonstrated. Independently of this paper, other recent work has developed several versions of the following result and applied it in various settings: if a deterministic rule can be OSP-implemented in pure strategies through a game form satisfying some notion of recall, then it can be OSP-implemented in pure strategies through a game form where agents take turns publicly announcing some of what they do and do not know (Ashlagi and Gonczarowski, 2016; Pycia and Troyan, 2017; Bade and Gonczarowski, 2017). In such a game form, an agent may not reveal everything he knows at once, but as the agents confide in one another turn after turn, group trust is slowly built in the sense that it eventually becomes apparent to all that it is safe to share the truth. We refer to these game forms as round table mechanisms. See Section 1.3 for a detailed comparison of our Proposition 2 to these other results.

Unfortunately, the above result is of limited use in the analysis of stochastic rules, where a group would like to condition a probabilistic distribution of outcomes on their collective information - for two reasons. First, the result only applies if the analyst restricts attention to game forms where the players' choices determine lotteries, and this modeling approach faces a conceptual issue: if a terminal history assigned a lottery is appropriately expanded into a history where the administrator randomly selects a terminal history assigned a sure outcome, then a strategy that was obviously dominant may no longer be. In other words, when a player contemplates what may happen when comparing strategies, he must consider randomization during play in a different manner from randomization after play. Second, even if this conceptual issue is set aside, the modeling approach requires all desired randomization to occur after play concludes instead of over the course of play, which is a serious restriction for OSP-implementation. This restriction is sharply illustrated with random serial dictatorship for the assignment of indivisible objects to agents: while this rule has a "random OSP-implementation" where the administrator randomizes at the start of play (Pycia and Troyan, 2017), it has no OSP-implementation where the desired lotteries are determined by play (Theorem 2). When investigating the OSP-implementation of stochastic rules, then, it is not safe to restrict attention to round table mechanisms.

Fortunately, there $i s$ in fact a canonical class to which the analyst can safely restrict attention for a formal notion of random OSP-implementation. In particular, our main result states that for the analysis of stochastic rules, it is safe to restrict attention to what we call randomized round table mechanisms, where the administrator acts once at the start of play, randomly selecting a round table mechanism (Theorem 1). Familiar examples of randomization at the start of a mechanism include the selection of a priority order for random serial dictatorship and the selection of school priorities for students in school choice. We emphasize that this revelation principle still requires the analyst to consider the entire class of randomized round table mechanisms, in contrast to the classic revelation principle for dominant strategy implementation, which allows the analyst to the focus on a single canonical game form; this point is also raised by Arribillaga, Massó, and Neme (2017).

The precise statement of our main result is particularly general; when applied to deterministic rules, it improves on the other revelation principles in several ways. Most notably, it has thus far not been known whether or not there are any rules that can only be OSP-implemented through game forms that violate recall requirements. In situations where players are individuals, such a game form might require someone to forget a previous action - or even to be unsure if he has already been called to play - and it perhaps 
goes without saying that the institutionalized altering of the contents of an individual's memory raises serious ethical (and practical!) concerns. On the other hand, in situations where players are groups (such as organizations, firms, or households), such a rule could be OSP-implemented if various representatives of a group are sometimes called to play without knowledge of whether or not other representatives have been called and how they have played - say, if the mechanism's administrator decides who to interview and what to ask based on previous interview responses (Isbell, 1957). Because we drop all information set restrictions, our result implies that there are no such rules: there is no need for technology that alters memories, and it is always sufficient to summon a single representative from each group to play. ${ }^{2}$

\subsection{Further results}

After establishing our revelation principle and the necessity of this generalization, we provide three further results related to OSP-implementation and canonical classes of mechanisms.

First, we generalize the model to accommodate randomization by the players in addition to the administrator. At first glance, it is ambiguous whether this would expand or shrink the collection of rules which can be OSP-implemented. On the one hand, perhaps more rules can be implemented simply because there are additional strategies that players have access to. On the other hand, perhaps fewer rules can be implemented: when an agent contemplates what might happen when comparing strategies, he has more to worry about when he and his opponents might randomize, reducing occurrences of obvious dominance. This latter point is especially a concern in game forms that violate recall assumptions: under the interpretation that representatives of a group are called to play, behavioral strategies allow a group to realize choices that, without randomization, would require coordination between representatives that is forbidden by the structure of the game form.

Unfortunately, our proof approach is not able to accommodate probabilistic notions of OSP-implementation, even if it is almost-sure (Example 1). That said, using a sure notion of OSP-implementation, we establish that if a deterministic rule has an OSPimplementation in any kind of strategies (pure, mixed, or behavioral), then it has an OSP-implementation in pure strategies through a round table mechanism - even if a group entertains the possibility that it, or its opponents, may later discover a way to coordinate choices in a manner that is forbidden by the laws of the mechanism (Theorem 3). Altogether, while player randomization does not help, the contemplation of player randomization does not hurt; in this way our reassurance that it is safe to restrict attention to round table mechanisms is particularly robust.

We then use our revelation principle to prove a result in the spirit of Carroll (2017) for settings where there is a finite collection of outcomes, and where agents have von Neumann-Morgenstern types compatible with strict rankings of outcomes. In particular, we prove that if a stochastic rule has an OSP-implementation, then it is ordinal, and moreover has a round table implementation where agents make public announcements simply about their rankings of deterministic outcomes (Theorem 4). Whereas the justi-

\footnotetext{
${ }^{2}$ Our main result also improves on previous revelation principles by using the largest class of game trees for which choices determine outcomes (Alós-Ferrer and Ritzberger, 2016); as a side contribution, we provide a new description of this class (Proposition 1).
} 
fication for ordinal mechanisms provided by Carroll (2017) relies on interdependence of agents' preferences over lotteries, ours does not due to our stronger solution concept.

Finally, we consider ordinary strategy-proofness (SP) in the context of round table mechanisms. As an immediate corollary to Theorem 2, if no round table mechanism provides an SP-implementation for a deterministic rule, then the rule has no OSPimplementation at all. Remarkably, the converse is true as well, providing a characterization of the deterministic rules with OSP-implementations: a deterministic rule has an OSP-implementation if and only if it has a SP-implementation through a round table mechanism (Theorem 5). ${ }^{3}$ Thus any investigation of OSP-implementation for a particular deterministic rule can be performed entirely using a familiar solution concept and a simple class of game forms.

\subsection{Related revelation principles}

In this section, we provide a detailed comparison of our Proposition 2 for deterministic rules to the other revelation principles in the literature.

A revelation principle takes the following form: if a rule can be implemented through a game form in class $\Gamma_{B I G}$, then it can be implemented through one in class $\Gamma_{S M A L L} \subseteq \Gamma_{B I G}$. Such a statement is made logically stronger when $\Gamma_{B I G}$ is made larger, as well as when $\Gamma_{S M A L L}$ is made smaller. Independently of this work, a series of closely-related revelation principles for obvious strategy-proofness were established in recent working papers (Ashlagi and Gonczarowski, 2016; Pycia and Troyan, 2017; Bade and Gonczarowski, 2017), and this section is dedicated to discussing the relationship between our revelation principle and these three. ${ }^{4}$ We remark that in each of these other papers, the revelation principle is not the primary contribution, but rather plays the role of a lemma toward the main result.

Ashlagi and Gonczarowski (2016) take $\Gamma_{B I G}$ to be the class of game forms with perfect recall, as in $\mathrm{Li}$ (2016), and first observe that it is without loss of generality to restrict attention to $\Gamma_{1}$, the class of game forms with perfect information where nature (alternatively, the mechanism's administrator) does not randomize.

Pycia and Troyan (2017) also take $\Gamma_{B I G}$ to be the class of game forms with perfect recall. Under preference restrictions that allow for a wide range of economic environments (such as object allocation), but rule out those with monetary transfers, they show that it is without loss of generality to restrict attention to $\Gamma_{2}$, their class of millipede mechanisms. These mechanisms have remarkable further structure: at any information set, an agent may or may not have one passing action, after which he may move again; each of his other actions is a clinching actions, guaranteeing that the outcome belongs to a particular one of his indifference classes, after which he does not move again.

Bade and Gonczarowski (2017) take $\Gamma_{B I G}$ to be the class of game forms for which no path from the root intersects the same information set twice; this class is larger than the class of game forms with perfect recall (because, for example, an agent may 'forget' the previous action he selected), but smaller than ours (because, for example, an agent may not 'forget' whether or not he has already selected an action). They show that it

${ }^{3} \mathrm{I}$ am indebted to Shengwu Li for conjecturing Theorem 5 to me through private correspondence.

${ }^{4}$ To my knowledge, Ashlagi and Gonczarowski (2016), Pycia and Troyan (2017), Bade and Gonczarowski (2017), and this paper were made publicly available in that order. I became aware of the other three papers after completing this paper. 
is without loss of generality to restrict attention to $\Gamma_{3}$, their class of gradual revelation mechanisms. These are our round table mechanisms with additional structure:

- no agent consecutively moves twice, or ever selects from a singleton set of actions, and

- whenever an agent's strategy guarantees (from some history) that all possible outcomes belong to the same indifference class regardless of his preference relation, the first action of that strategy is to publicly announce his preference relation (and take no further action thereafter).

Because $\Gamma_{3}$ is a subset of our class of round table mechanisms, the result of Bade and Gonczarowski (2017) can be used to immediately strengthen our main result; we do not do so in the body of this paper in the interest of clarity about credit among recent working papers.

With respect to these other revelation principles, our contribution is not to further shrink $\Gamma_{S M A L L}$, but rather to enlarge $\Gamma_{B I G}$. In particular, we drop all recall requirements and work with the largest class of game trees for which choices determine plays.

\section{Base Model}

\section{$2.1 \quad$ Overview}

A group of agents $N=\{1,2, \ldots, n\}$ face a set of (public) outcomes $X$. Each agent $i$ has private information summarized by his type $\theta_{i} \in \Theta_{i}$, which determines his (complete and transitive) preference relation $R_{\theta_{i}}$ ranking outcomes. Critically, an agent's ranking of outcomes is fixed; it would not change were he to learn anything about his peers' types, ruling out for example auctions for mineral rights (Milgrom and Weber, 1982). A type profile $\theta=\left(\theta_{i}\right)_{i \in N}$ specifies a type for each agent, and the set of possible type profiles is given by $\Theta \equiv \times_{i \in N} \Theta_{i}$.

In our base model, the agents wish to condition the outcome selection on their collective information according to some deterministic rule $f: \Theta \rightarrow X$. Because the type profile is not common knowledge, their objective is to weakly implement $f$ according to a particular solution concept using a game form (or mechanism).

In this section, we introduce the base model for deterministic rules and pure strategies. We begin with our class of mechanisms; describing the associated class of game trees involves a digression on order theory (Section 2.2). We then introduce the solution concept of obvious strategy-proofness for pure strategies (Section 2.3). Finally, we describe when a mechanism implements a deterministic rule, in the sense that the rule's recommendation is plausibly reached no matter the type profile (Section 2.4). Our presentation of these notions in this section allows us to then simply describe each of our main results using an appropriate extension of the base model.

\subsection{Extensive game forms}

To implement the rule, we consider extensive game forms (Osborne and Rubinstein, 1994; $\mathrm{Li}, 2016)$. Intuitively, this is an extensive-form game tree without preference information, which becomes an extensive-form game when paired with (the preference profile associated with) a type profile. 
An extensive game form involves a game tree, which in its most general formulation is a collection of histories $H$ that is partially ordered by precedence $\precsim$. So that our revelation principle may be compatible with as many game trees as possible, we begin by reviewing some concepts from order theory. For a fixed set $H$, a partial order $\precsim$ is a binary relation that is reflexive, antisymmetric, and transitive, but not necessarily complete; we denote the associated strict order by $\prec$. For each pair $h, h^{\prime} \in H$, if $h \prec h^{\prime}$ and there is no $h^{\prime \prime}$ such that $h \prec h^{\prime \prime} \prec h^{\prime}$, then we say both that

(i) $h$ is an immediate predecessor of $h^{\prime}$, and

(ii) $h^{\prime}$ is an immediate successor of $h$.

A subset $H^{\prime} \subseteq H$ is a chain if for each pair $h, h^{\prime} \in H^{\prime}$, either $h \precsim h^{\prime}$ or $h^{\prime} \precsim h$; a chain $H^{\prime}$ is maximal if there is no chain $H^{\prime \prime}$ such that $H^{\prime} \subsetneq H^{\prime \prime}$; a subset $H^{\prime} \subseteq H$ is well-ordered if for each $H^{\prime \prime} \subseteq H^{\prime}, H^{\prime \prime}$ has a minimum (in which case $H^{\prime}$ is a chain). For each pair $h, h^{\prime} \in H$, the meet of $h$ and $h^{\prime}, h \wedge h^{\prime} \in H$, is the greatest lower bound of $\left\{h, h^{\prime}\right\}$, which is unique if it exists.

Definition: A meet-semilattice is a partially ordered set $(H, \precsim)$ such that for each pair $h, h^{\prime} \in H$, there is $h \wedge h^{\prime}$.

Definition: A tree (see the Jech, 1971 survey) is a partially ordered set $(H, \precsim)$ such that for each $h \in H,\left\{h^{\prime} \in H \mid h^{\prime} \precsim h\right\}$ is well-ordered. It is rooted if there is $\min H$.

For our purposes, a maximal chain is a play, or a complete description of a sequence of choices, and a game tree is "admissible" if choices always induce a unique play. This notion is made precise in Alós-Ferrer and Ritzberger (2016), and we refer the reader there for the precise definition. The result that interests us here is the following:

Theorem (Alós-Ferrer And Ritzberger, 2016): ${ }^{5}$ A rooted "pseudotree" is "admissible" if and only if it satisfies "weak up-discreteness," "coherence," and "regularity."

We use this alternative statement involving notions we find more familiar:

Proposition 1: The class of meet-semilattice trees is the class of "admissible" rooted "pseudotrees."

The proof is in Appendix A. When describing these game forms, it is convenient to view the (mechanism) administrator as player 0 and write $N_{0} \equiv N \cup\{0\}$ for the set of agents together with the administrator. The administrator publicly commits to how he will behave in advance, playing the same role as the 'chance player' or 'nature' in the literature on games. Following Aumann (1964), we include how choices can be randomized in our description; we here include only states for the administrator, and later extend the base model with different kinds of states for the agents. Formally:

Definition: An extensive game form is given by a tuple

$$
\left\langle H, \precsim, A, \mathcal{A}, P,\left(\mathcal{I}_{i}\right)_{i \in N}, g,\left(\Omega_{h}\right)_{h \in H_{0}},\left(B_{h}\right)_{h \in H_{0}}\right\rangle \text {, where }
$$

\footnotetext{
${ }^{5}$ This is first proven in Alós-Ferrer and Ritzberger (2008) with a minor error, which is corrected in Alós-Ferrer, Kern, and Ritzberger (2011). We refer the reader to Alós-Ferrer and Ritzberger (2016) for this result's most complete treatment.
} 
1. $H$ is the set of histories and $\precsim$ is the partial order on $H$ representing precedence. We require that $(H, \precsim)$ is a meet-semilattice tree. These conditions guarantee that choices always determine a unique play, guarantee that there is a unique initial history $h_{\emptyset}$ which precedes all others, and allow an action to be viewed as the selection of an immediate successor. We let $\sigma(h)$ denote the immediate successors of $h$. A play is a maximal chain, which gives a complete description of a sequence of choices; we write $\pi$ for a play and $\Pi$ for the set of plays. A terminal history is a history with no successor; we write $z$ for a terminal history and $Z$ for the (possibly empty) set of terminal histories.

2. $A$ is the set of actions and $\mathcal{A}: \cup_{H} \sigma(h) \rightarrow A$ is the action function, which at each history $h$ associates each immediate successor $h^{\prime} \in \sigma(h)$ with the action taken to reach it. We require that at any history, each available action determines a unique next history: for each $h \in H$ and each pair $h^{\prime}, h^{\prime \prime} \in \sigma(h), \mathcal{A}\left(h^{\prime}\right) \neq \mathcal{A}\left(h^{\prime \prime}\right)$. For each history $h$, we let $A(h) \equiv \cup_{h^{\prime} \in \sigma(h)} \mathcal{A}\left(h^{\prime}\right)$ denote the actions available at $h$.

3. $P: H \backslash Z \rightarrow N_{0}$ is the player function, which associates each non-terminal history with whoever selects an action at that history - either an agent or the administrator. For each $i \in N_{0}$, we let $H_{i} \equiv\{h \in H \mid P(h)=i\}$ denote the histories that belong to $i$. Similarly $H_{N} \equiv\{h \in H \mid P(h) \in N\}$.

4. for each $i \in N, \mathcal{I}_{i}$ is the information partition for $i$, which specifies the information sets partitioning $H_{i}$. We require that for each pair $h, h^{\prime}$ in the same information set $I_{i}$, the same actions are available: $A(h)=A\left(h^{\prime}\right)$. We write $A\left(I_{i}\right)$ for the actions $A(h)$ available at each history $h \in I_{i}$. Across all histories in a given information set $I_{i}, i$ must behave the same way. Implicitly, the administrator's information set $\mathcal{I}_{0}$ consists only of singletons.

5. $g: \Pi \rightarrow X$ is the outcome function, which associates each play with an outcome.

6. at each $h \in H_{0}$, there is a set of states $\Omega_{h}$ with which the administrator can condition his action at $h$, and the administrator commits to $B_{h}: \Omega_{h} \rightarrow A(h)$ specifying how he will condition his act on the state's realization. In other words, the administrator commits to a behavioral strategy.

Let $\Gamma$ denote the class of extensive game forms (with agents in $N$ and outcomes in $X$ ).

\subsection{Solution Concept}

For the rest of this section, fix an extensive game form $G$. While an agent is restricted to pure strategies, he may worry that his opponents are somehow able to avoid this restriction. We therefore also introduce "choice functions" that agents are forbidden from selecting; these can be thought of as realizations of behavioral strategies that are not necessarily pure strategies.

Definition: Kinds of strategies for agent $i$.

- a choice function for $i$ is a mapping $C_{i}$ which associates each history $h \in H_{i}$ with an available action $C(h) \in A(h)$. We use $\mathcal{C}_{i}$ for the set of choice functions of $i, \mathcal{C}$ for the set of choice function profiles, and $\mathcal{C}_{-i}$ for the set of lists of choice functions from the agents other than $i$. 
- a pure strategy for $i$ is a choice function for $i$ with the restriction that if $h$ and $h^{\prime}$ share an information set, then $S(h)=S\left(h^{\prime}\right)$. We use $\mathcal{S}_{i}$ for the set of pure strategies of $i, \mathcal{S}$ for the set of pure strategy profiles, and $\mathcal{S}_{-i}$ for the set of lists of pure strategies from the agents other than $i$.

A solution concept specifies a collection of strategy profiles for each game - in practice, typically the strategy profiles that are deemed 'plausible' in some particular sense. Here, we are only interested in games formed by pairing some extensive game form with (the preference profile associated with) a type profile: the domain is $\Gamma \times \Theta$, and each $(G, \theta)$ is associated with a collection in $2^{\mathcal{S}}$.

Our solution concept involves the repeated comparison of one's strategy to alternatives over the course of play. To speak of strategy evaluation at a given information set, we write $\pi^{G}\left(h, C, C_{0}\right)$, or sometimes $\pi^{G}\left(h, C_{i}, C_{-i}, C_{0}\right)$, for the resulting play when we start from $h$ and play proceeds according to $\left(C, C_{0}\right)$, and we write $x^{G}\left(h, C, C_{0}\right)$ for the associated outcome $g\left(\pi^{G}\left(h, C, C_{0}\right)\right)$. These functions are well-defined (see Alós-Ferrer and Ritzberger, 2016 and Appendix A). For each choice function $C_{i} \in \mathcal{C}_{i}$, define the reachable histories for $C_{i}$ and the reachable information sets for $C_{i}$ by:

$$
\begin{aligned}
H_{i}\left(C_{i}\right) & \equiv\left\{h \in H_{i} \mid \text { there are } C_{-i}, C_{0} \text { such that } h \in \pi^{G}\left(h_{\emptyset}, C_{i}, C_{-i}, C_{0}\right)\right\}, \text { and } \\
\mathcal{I}_{i}\left(C_{i}\right) & \equiv\left\{I_{i} \in \mathcal{I}_{i} \mid \text { there is } h \in I_{i} \text { such that } h \in H_{i}\left(C_{i}\right)\right\} .
\end{aligned}
$$

In particular, $\mathcal{I}_{i}\left(C_{i}\right)$ is the collection of information sets where $i$ could possibly find himself if he has been playing according to $C_{i}$, provided that any peer could realize any choice function through a behavioral strategy. We use the notation $\mathcal{I}_{i}\left(S_{i}\right)$ when the choice function is a pure strategy.

Definition: Obviously dominant strategies for a fixed game $(G, \theta)$.

- A pure strategy $S_{i} \in \mathcal{S}_{i}$ is obviously $\mathcal{S}$-dominant if for each $I_{i} \in \mathcal{I}_{i}\left(S_{i}\right)$, each pair $h, h^{\prime} \in I_{i}$, each $S_{i}^{\prime} \in \mathcal{S}_{i}$ such that $S_{i}\left(I_{i}\right) \neq S_{i}^{\prime}\left(I_{i}\right),{ }^{6}$ each pair $S_{-i}, S_{-i}^{\prime} \in \mathcal{S}_{-i}$, and each pair $\omega_{H_{0}}, \omega_{H_{0}}^{\prime} \in \Omega_{H_{0}}$,

$$
\begin{gathered}
x^{G}\left(h, S_{i}, S_{-i}, B_{0}\left(\omega_{H_{0}}\right)\right) \\
R_{i} \\
x^{G}\left(h^{\prime}, S_{i}^{\prime}, S_{-i}^{\prime}, B_{0}\left(\omega_{H_{0}}^{\prime}\right)\right) .
\end{gathered}
$$

- A pure strategy $S_{i} \in \mathcal{S}_{i}$ is obviously $\mathcal{C}$-dominant if for each $I_{i} \in \mathcal{I}_{i}\left(S_{i}\right)$, each pair $h, h^{\prime} \in I_{i}$, each $C_{i}^{\prime} \in \mathcal{C}_{i}$ such that $S_{i}\left(I_{i}\right) \neq C_{i}^{\prime}\left(I_{i}\right)$, each pair $C_{-i}, C_{-i}^{\prime} \in \mathcal{C}_{-i}$, and each pair $C_{0}, C_{0}^{\prime} \in \mathcal{C}_{0}$,

$$
\begin{gathered}
x^{G}\left(h, S_{i}, C_{-i}, C_{0}\right) \\
R_{i} \\
x^{G}\left(h^{\prime}, C_{i}^{\prime}, C_{-i}^{\prime}, C_{0}^{\prime}\right) .
\end{gathered}
$$

${ }^{6}$ In the original definition (Li, 2016), $S_{i}$ need only be compared to $S_{i}^{\prime}$ if moreover $S_{i}^{\prime}$ can reach $I_{i}$. The two definitions are not equivalent when there is absent-mindedness, but the results in this paper hold for both definitions, essentially because any additional paths from $I_{i}$ that must be considered under the given definition can never be reached and can thus be safely pruned from the game tree. We work with the given definition for its conceptual simplicity: at a given information set, the agent considers switching to any strategy that prescribes a different action. 
In order for a pure strategy $S_{i}$ to be obviously $\mathcal{S}$-dominant, it must pass a series of comparisons at each reachable information set. In particular, at each reachable information set, $S_{i}$ must be compared to each pure strategy $S_{i}^{\prime}$ that prescribes a different action. In order for $S_{i}$ to pass this comparison, each possible outcome under $S_{i}$ must be at least as desirable as each possible outcome under $S_{i}^{\prime}$.

The latter notion is much stronger: if a pure strategy is obviously $\mathcal{C}$-dominant, then not only is it obviously $\mathcal{S}$-dominant, but moreover, the agent entertains the possibilities that:

- he may later discover a way to coordinate across histories that share an information set,

- his opponents may later discover a way to coordinate across histories that share an information set, and

- the administrator may deviate from his announced behavioral strategy.

Under the interpretation that each player is a group with different representatives making choices at different histories (Isbell, 1957), obvious $\mathcal{C}$-dominance requires each representative to contemplate the possibility that representatives of its group, or another group, may discover a way to illegally coordinate.

Our solution concept is that each agent plays an obviously dominant strategy.

Definition: Obvious strategy-proofness.

- A pure strategy profile $S$ belongs to $\operatorname{OSP}^{\mathcal{S}}(G, \theta)$ if and only if for each agent $i, S_{i}$ is obviously $\mathcal{S}$-dominant.

- A pure strategy profile $S$ belongs to $\operatorname{OSP}^{\mathcal{C}}(G, \theta)$ if and only if for each agent $i, S_{i}$ is obviously $\mathcal{C}$-dominant.

\subsection{Implementation}

A game form is a game with incomplete information; in particular, preference information is missing. In the context of a mechanism design model, we can say more specifically that the type profile is unknown. Following Harsanyi (1967), suppose the administrator imagines that each agent $i$ is drawn from a population partitioned by types in $\Theta_{i}$, and considers one plausible strategy for each type class. A Harsanyi pure strategy is a mapping $\mathbb{S}_{i}: \Theta_{i} \rightarrow \mathcal{S}_{i}$

Informally, we say that a game form implements a rule if there is some Harsanyi strategy profile such that no matter how the administrator plays, for each type profile, the associated strategy profile (i) is plausible according to our solution concept, and (ii) yields the rule's recommendation. Formally:

Definition: Obvious strategy-proof implementation.

- A deterministic rule $f$ is $\mathbf{O S P}^{\mathcal{S}}$-implementable if there are

(i) $G \in \Gamma$, and

(ii) an associated Harsanyi pure strategy profile $\mathbb{S}$,

such that for each $\theta \in \Theta$, 
(i) $\left(\mathbb{S}_{i}\left(\theta_{i}\right)\right) \in \operatorname{OSP}^{\mathcal{S}}(G, \theta)$, and

(ii) for each $\omega_{H_{0}} \in \Omega_{H_{0}}$,

$$
x^{G}\left(h_{\emptyset},\left(\mathbb{S}_{i}\left(\theta_{i}\right)\right)_{i \in N}, B_{0}\left(\omega_{H_{0}}\right)\right)=f(\theta) .
$$

In this case we say $G \mathbf{O S P}^{\mathcal{S}}$-implements $f$ through $\mathbb{S}$.

- A deterministic rule $f$ is $\mathbf{O S P}^{\mathcal{C}}$-implementable if there are

(i) $G \in \Gamma$, and

(ii) an associated Harsanyi pure strategy profile $\mathbb{S}$, such that for each $\theta \in \Theta$,

(i) $\left(\mathbb{S}_{i}\left(\theta_{i}\right)\right) \in \operatorname{OSP}^{\mathcal{C}}(G, \theta)$, and

(ii) for each $\omega_{H_{0}} \in \Omega_{H_{0}}$,

$$
x^{G}\left(h_{\emptyset},\left(\mathbb{S}_{i}\left(\theta_{i}\right)\right)_{i \in N}, B_{0}\left(\omega_{H_{0}}\right)\right)=f(\theta) .
$$

In this case we say $G \mathbf{O S P}^{\mathcal{C}}$-implements $f$ through $\mathbb{S}$.

Of these two implementation notions, the latter is stronger.

\subsection{Round table mechanisms and base revelation principle}

The revelation principle for deterministic rules involves the following canonical class of mechanisms:

Definition: A round table mechanism is a perfect-information game form such that each non-terminal history $h$ belongs to some agent $i \in N$. Moreover, actions in $A(h)$ are subsets of $\Theta_{i}$, and any two actions in $A(h)$ are pairwise disjoint. At any history of $i$ with no strict predecessor belonging to $i$, the union of actions in $A(h)$ is $\Theta_{i}$; at any other history $h$ of $i$, the union of actions in $A(h)$ is the intersection of the actions at all predecessors that lead to $h$. The class of round table mechanisms is denoted $\Gamma^{R T}$.

Definition: A deterministic rule has a round table implementation if there is $G \in \Gamma^{R T}$ such that $G \mathbf{O S P}^{\mathcal{S}}$-implements $f$ through $\mathbb{S}$, where for each $i \in N$, each $\theta_{i} \in \Theta_{i}$, and each $h \in H_{i}\left(\mathbb{S}_{i}\left(\theta_{i}\right)\right), \mathbb{S}_{i}\left(\theta_{i}\right)(h)$ is the unique action solving

$$
\theta_{i} \in \mathbb{S}_{i}\left(\theta_{i}\right)(h)
$$

We note that $\mathbf{O S P}^{\mathcal{S}}$-implementation with a round table mechanism implies $\mathbf{O S P}^{\mathcal{C}}$ implementation. The logic is simple: in a round table mechanism, each choice function is a pure strategy.

Our statement of the revelation principle for deterministic rules improves on other statements by allowing all game forms for which choices determine plays and removing all recall assumptions:

Proposition 2: If a deterministic rule has an $\operatorname{OSP}^{\mathcal{S}}$-implementation, then it has a round table implementation, which is an $\mathbf{O S P}^{\mathcal{C}}$-implementation. 
The proof is in Appendix B. This result is both a lemma for, and a corollary to, two of our main results: Theorem 1 for implementation of stochastic rules and Theorem 3 for robustness to player randomization.

\section{Main Results}

\subsection{Revelation principle for stochastic rules}

Our main result generalizes Proposition 2 to the case where agents wish to condition a probabilistic distribution of outcomes on their collective information. A key step in this generalization is the elegant argument in Lemma 2 of Pycia and Troyan (2017) that all of the administrator's randomization can be done at the initial history; we provide this argument in our proof with some additional formality.

In order to pursue stochastic rules, we equip the set of outcomes $X$ with a $\sigma$-algebra $\mathcal{X} \subseteq 2^{X}$ of measurable collections of outcomes. A lottery is a probability measure $p: \mathcal{X} \rightarrow$ $[0,1]$, and we denote the set of lotteries by $\mathcal{P}$. A stochastic rule is a function $f: \Theta \rightarrow \mathcal{P}$.

We consider only pure strategies for the implementation of stochastic rules, as issues quickly arise when the agents themselves randomize (see Example 1 in Section 3.3). To extend the base model, the minimal additional structure we require is a $\sigma$-algebra $\mathcal{A}$ on $\Omega_{H_{0}}$ and a probability measure $\mu: \mathcal{A} \rightarrow[0,1]$, allowing the assignment of probabilities to measurable events upon which the administrator conditions. Let us call an extensive game form together with this additional structure $(G, \mathcal{A}, \mu)$ a minimally-probabilistic extensive game form, and let $\Gamma^{M P}$ denote their class. We adapt our implementation notion as follows:

DeFINITION: Random obvious strategy-proof implementation.

- A stochastic rule $f$ is random $\mathbf{O S P}^{\mathcal{S}}$-implementable if there are

(i) $(G, \mathcal{A}, \mu) \in \Gamma^{M P}$, and

(ii) an associated Harsanyi pure strategy profile $\mathbb{S}$,

such that for each $\theta \in \Theta$,

(i) $\left(\mathbb{S}_{i}\left(\theta_{i}\right)\right) \in \operatorname{OSP}^{\mathcal{S}}(G, \theta)$, and

(ii) for each $X^{\prime} \in \mathcal{X}$,

$$
\begin{aligned}
& \left\{\omega_{H_{0}} \in \Omega_{H_{0}} \mid x^{G}\left(h_{\emptyset},\left(\mathbb{S}_{i}\left(\theta_{i}\right)\right)_{i \in N}, B_{0}\left(\omega_{H_{0}}\right)\right) \in X^{\prime}\right\} \in \mathcal{A}, \text { and } \\
& \mu\left(\left\{\omega_{H_{0}} \in \Omega_{H_{0}} \mid x^{G}\left(h_{\emptyset},\left(\mathbb{S}_{i}\left(\theta_{i}\right)\right)_{i \in N}, B_{0}\left(\omega_{H_{0}}\right)\right) \in X^{\prime}\right\}\right)=f(\theta)\left(X^{\prime}\right) .
\end{aligned}
$$

In this case we say $G$ random $\mathbf{O S P}^{\mathcal{S}}$-implements $f$ through $\mathbb{S}$.

- A stochastic rule $f$ is random $\mathbf{O S P}^{\mathcal{C}}$-implementable if there are

(i) $(G, \mathcal{A}, \mu) \in \Gamma^{M P}$, and

(ii) an associated Harsanyi pure strategy profile $\mathbb{S}$,

such that for each $\theta \in \Theta$,

(i) $\left(\mathbb{S}_{i}\left(\theta_{i}\right)\right) \in \operatorname{OSP}^{\mathcal{C}}(G, \theta)$, and

(ii) for each $X^{\prime} \in \mathcal{X}$,

$$
\left\{\omega_{H_{0}} \in \Omega_{H_{0}} \mid x^{G}\left(h_{\emptyset},\left(\mathbb{S}_{i}\left(\theta_{i}\right)\right)_{i \in N}, B_{0}\left(\omega_{H_{0}}\right)\right) \in X^{\prime}\right\} \in \mathcal{A} \text {, and }
$$




$$
\mu\left(\left\{\omega_{H_{0}} \in \Omega_{H_{0}} \mid x^{G}\left(h_{\emptyset},\left(\mathbb{S}_{i}\left(\theta_{i}\right)\right)_{i \in N}, B_{0}\left(\omega_{H_{0}}\right)\right) \in X^{\prime}\right\}\right)=f(\theta)\left(X^{\prime}\right) .
$$

In this case we say $G$ random $\mathbf{O S P}^{\mathcal{C}}$-implements $f$ through $\mathbb{S}$.

We note that it is sometimes possible to represent the probabilities specified by $\mu$ as calculations involving independent probabilities across different histories in $H_{0}$, for example when each of the administrator's state spaces is the unit interval together with the Lebesgue measurable sets and the Lebesgue measure (Aumann, 1964). Our approach does not require any such restrictions, and even allows the administrator to correlate his choices across histories. Finally, we remark that as long as values of $\mu$ can be assigned to the events specified above, a suitable $\mathcal{A}$ can be recovered. ${ }^{7}$

We emphasize that this notion of random implementation does not require lotteries to be assigned to terminal nodes. Given such a game form, one could replace each terminal node with a move by the administrator where he randomly selects an outcome according to the associated lottery. One could also consider game forms where the administrator randomizes at various histories scattered throughout the game tree. In each of these cases, it is possible for a stochastic rule to be implemented.

In our canonical class of mechanisms, the administrator moves once and only once, beginning play by selecting a round table mechanism for the agents:

Definition: A randomized round table mechanism is a perfect-information minimallyprobabilistic extensive game form such that the initial history belongs to the administrator 0 , and every other non-terminal history $h$ belongs to some agent $i \in N$. Moreover, at each history $h$ of player $i$, actions in $A(h)$ are subsets of $\Theta_{i}$, and any two actions in $A(h)$ are pairwise disjoint. At any history of $i$ with no strict predecessor belonging to $i$, the union of actions in $A(h)$ is $\Theta_{i}$; at any other history $h$ of $i$, the union of actions in $A(h)$ is the intersection of the actions at all predecessors that lead to $h$. The class of randomized round table mechanisms is denoted $\Gamma^{R R T}$.

DEFINITION: A stochastic rule has a randomized round table implementation if there is $(G, \mathcal{A}, \mu) \in \Gamma^{R R T}$ such that $(G, \mathcal{A}, \mu) \mathbf{O S P}^{\mathcal{S}}$-implements $f$ through $\mathbb{S}$, where for each $i \in N$, each $\theta_{i} \in \Theta_{i}$, and each $h \in H_{i}\left(\mathbb{S}_{i}\left(\theta_{i}\right)\right), \mathbb{S}_{i}\left(\theta_{i}\right)(h)$ is the unique action solving

$$
\theta_{i} \in \mathbb{S}_{i}\left(\theta_{i}\right)(h)
$$

We note that random $\mathbf{O S P}^{\mathcal{S}}$-implementation with a randomized round table mechanism implies random $\mathbf{O S P}^{\mathcal{C}}$-implementation: each choice function is a pure strategy, and the administrator is done playing at each player history. Our main result is that it is safe to restrict attention to this class:

THEOREM 1: If a stochastic rule has a random $\mathbf{O S P}^{\mathcal{S}}$-implementation, then it has a randomized round table implementation, which is a random $\mathbf{O S P}^{\mathcal{C}}$-implementation.

\footnotetext{
${ }^{7}$ To see this, for each $\theta \in \Theta$, consider the mapping $x_{\theta}^{G}: \Omega_{H_{0}} \rightarrow X$ that takes each state profile $\omega_{H_{0}}$ to the outcome $x^{G}\left(h_{\emptyset},\left(\mathbb{S}_{i}\left(\theta_{i}\right)\right)_{i \in N}, B_{0}\left(\omega_{H_{0}}\right)\right)$. Because $\mathcal{X}$ is a $\sigma$-algebra, it is well known that the inverse images of $x_{\theta}^{G}$ form a $\sigma$-algebra $\mathcal{A}_{\theta}$. Moreover, it is well-known that an arbitrary intersection of $\sigma$-algebras is itself a $\sigma$-algebra; thus $\mathcal{A}$ may be taken to be $\cap \mathcal{A}_{\theta}$. It is straightforward to complete the measure $\mu$ from the given probabilities.
} 
The proof is in Appendix C. In the next section, we establish that this generalization is necessary, in the sense that it is not safe to restrict attention to the canonical class for deterministic rules.

\subsection{Insufficiency of round table mechanisms for stochastic rules}

In this section, we demonstrate by means of a simple example that Proposition 2 should not be used when investigating the implementation of stochastic rules.

There is a set of agents $N=\{1,2,3\}$ with generic members $i, j, k$ and a set of objects $\{A, B, C\}$ with generic members $a, b, c$. An allocation is a bijection between the agents and objects, associating each agent with his own object, and an outcome in $X$ is a lottery over allocations. For each $i \in N, \Theta_{i}$ is the set of von Neumann-Morgenstern utility representations of preferences over lotteries of objects; as there is no indifference, we normalize so that for each $\theta_{i}$, there is some $m$ satisfying $9>m>0$ such that the range of $\theta_{i}$ is $\{0, m, 9\}$.

Definition: For each strict order of agents $i$ then $j$ then $k$, the rule $f^{i, j, k}$ gives $i$ his favorite object, $j$ his favorite of the other two objects, and $k$ the remaining object. The random serial dictatorship $f$ is given by the uniform randomization over these rules:

$$
f \equiv \frac{1}{6} \sum f^{i, j, k}
$$

THEOREM 2: Random serial dictatorship has no round table implementation.

The proof is in Appendix D. When paired with the observation by Pycia and Troyan (2017) that random serial dictatorship does have a randomized round table implementation, Theorem 2 establishes that Theorem 1 is a necessary generalization of Proposition 2 for the implementation of stochastic rules.

\subsection{Robustness to player randomization}

Does our revelation principle hold if, as assumed throughout most of game theory, the players might randomize? The answer depends on how this question is made precise, which involves a series of subtle modeling choices. We proceed briefly through the same steps from Section 3, occasionally pausing to highlight a choice of conceptual interest.

First of all, how should we model player randomization? Though for game forms with perfect recall, behavioral strategies are equivalent to mixed strategies (Kuhn, 1953; Aumann, 1964), this is not true for all game forms we consider; we therefore consider both. For our result, we are able to accommodate unusually general notions of randomization that do not require probabilities:

- For mixed strategies, let us say a mixed extensive game form $\left(G,\left(\Omega_{i}\right)_{i \in N}\right)$ consists of (i) an extensive game form $G$, and (ii) for each $i \in N$, a set of states $\Omega_{i}$ with which the player can condition his pure strategy in a mixed strategy. A mixed strategy for $i$ is a mapping $M_{i}: \Omega_{i} \rightarrow \mathcal{S}_{i}$ specifying how $i$ conditions his selection of pure strategy on the realization of a state at the start of the game. We use $\mathcal{M}_{i}, \mathcal{M}$, and $\mathcal{M}_{-i}$ to denote, respectively, the set of mixed strategies of $i$, the set of mixed strategy profiles, and the set of lists of mixed strategies from agents other than $i$. 
- For behavioral strategies, let us say a behavioral extensive game form $\left(G,\left(\Omega_{h}\right)_{h \in H_{N}}\right)$ consists of (i) an extensive game form $G$, and (ii) at each $h \in H_{N}$, a set of states $\Omega_{h}$ with which the player at $h$ can condition his action at $h$ in a behavioral strategy. We require that for each pair $h, h^{\prime}$ in the same information set, $\Omega_{h}=\Omega_{h^{\prime}}$; each is a copy of the other, though their realizations may be different. Thus at a given information set, the agent cannot infer anything about his history simply by looking at his state space. A behavioral strategy for $i$ is a list of mappings $B=\left(B_{h}\right)_{h \in H_{i}}$, where $B_{h}: \Omega_{h} \rightarrow A(h)$ specifies how $i$ conditions his selection of an action at $h$ on the realization of a state at $h$, with the restriction that if $h$ and $h^{\prime}$ share an information set, then $B_{h}=B_{h^{\prime}}$. We use $\mathcal{B}_{i}, \mathcal{B}$, and $\mathcal{B}_{-i}$ to denote, respectively, the set of mixed strategies of $i$, the set of mixed strategy profiles, and the set of lists of mixed strategies from agents other than $i$.

While we do not require randomization to involve probabilities, the addition of further familiar structure would of course not impact our results. ${ }^{8}$

In order to specify where an agent assesses a given strategy, we choose notions of reachable information sets $\mathcal{I}_{i}\left(M_{i}\right)$ and $\mathcal{I}_{i}\left(B_{i}\right)$ that are analogous to our original definition, simply modified to allow any realization of agent $i$ 's states. The more interesting question is when an agent assesses his strategy at a given information set: before or after states are realized?

There is a subtle conceptual problem with taking an ex-ante approach, which is best illustrated with behavioral strategies. At a given information set, suppose $B_{i}$ and $B_{i}^{\prime}$ prescribe the same range of outcomes. Before the state is realized, there is a straightforward process for determining whether or not the two are equivalent: for each state, check whether or not $B_{i}$ and $B_{i}^{\prime}$ prescribe the same action; if they agree at every state, they are equivalent; otherwise, they are distinct. The problem is that this process involves state-by-state contingent reasoning, and one of the primary motivations for obvious strategy-proofness is the observation that subjects may have difficulty with such reasoning ( $\mathrm{Li}, 2016)$. An agent without this ability who is tasked with making comparisons before the state is realized would therefore either have to (i) compare a strategy to itself or (ii) never compare a strategy to another that prescribes the same range of actions. We believe that neither of these alternatives is in the spirit of the original notion, and therefore use the following ex-post notions:

- For obvious $\mathcal{M}$-dominance, we assume that before the game begins, the state $\omega_{i}$ is revealed to the agent, and this information is recorded and available thereafter. At any given information set, then, each mixed strategy is already reduced to a pure strategy.

- For obvious $\mathcal{B}$-dominance, we do not assume that the agent has a record of every state that is revealed to him over the course of the game; indeed, without perfect recall, the agent may not even have a record of every action that he has taken. At

${ }^{8}$ For example, we could require for each state space: (i) a collection of events whose relative likelihood can be compared; (ii) that this collection is a $\sigma$-algebra; (iii) that each agent's likelihood comparisons have representation by a probability measure; and (iv) that these probability measures all correspond to some 'objective' measure. To guarantee that conditional probabilities can be defined and used to calculate lotteries of outcomes, we could impose that these probability spaces are standard (Mackey, $1957)$, provide $\sigma$-algebras for acts and outcomes, and impose measurability restrictions on mappings (Halmos, 1950; Aumann, 1964). To rule out strategy correlation (Aumann, 1987), we could impose that the product state spaces are associated with their product $\sigma$-algebras and product measures. 
a given information set, however, the current state $\omega_{x}$ is revealed, and the agent takes this information into account when making comparisons - though he does not know which of his information set's histories he is playing from, and therefore does not know which of these histories $\omega_{x}$ belongs to.

For brevity, we omit the formal definitions of obvious $\mathcal{M}$-dominance and obvious $\mathcal{B}$ dominance; these notions should be clear given this discussion and the analogous definition for pure strategies. The solution concepts $\operatorname{OSP}^{\mathcal{M}}(G, \theta)$ and $\operatorname{OSP}^{\mathcal{B}}(G, \theta)$, for mixed and behavioral strategies, respectively, are also analogous to the definition for pure strategies.

We use $\mathbb{M}_{i}: \theta_{i} \rightarrow \mathcal{M}_{i}$ for a Harsanyi mixed strategy and $\mathbb{B}_{i}: \theta_{i} \rightarrow \mathcal{B}_{i}$ for a Harsanyi behavioral strategy. Informally, we say that a game form implements a rule if there is some Harsanyi strategy profile such that, for each type profile, the associated strategy profile (i) is plausible according to our solution concept, and (ii) yields the rule's recommendation. There is one last subtle point that still must be addressed, however. Suppose the mechanism administrator wishes to implement a deterministic rule, yet he and the agents may randomize. When should we say he has succeeded? There are two natural definitions:

1. for each $\theta \in \Theta$, the outcome is $f(\theta)$ for each joint realization of states, or

2. for each $\theta \in \Theta$, the outcome is $f(\theta)$ with probability 1 .

Under the second notion, we are unable pursue our approach for our revelation principle, as we cannot replace any randomized strategy with any of its realizations:

EXAMPLE 1: There is one agent with one possible type, and there are two outcomes, $x$ and $x^{\prime}$. The agent is indifferent between these outcomes, and the rule specifies that $x$ should be selected. In the extensive game form $G$, the agent first selects a number from the unit interval, then does so once more, at which point the game ends. Moreover, the agent is absent-minded; in particular, all histories share an information set. The outcome function assigns $x^{\prime}$ only when the same number is selected twice, and assigns $x$ otherwise. The behavioral strategy $B$ selects a number from the unit interval using the uniform distribution at the sole information set. Though $(G, B)$ yields outcome $x$ with probability 1 , for each pure strategy $S$ and each mixed strategy $M,(G, S)$ and $(G, M)$ both yield outcome $x^{\prime}$ with probability 1 .

We therefore use the first notion, which is stronger and allows us to use a lessstructured model. Thus our implementation notion is not that the rule's recommendation is selected almost surely, but rather that it is selected surely-for every realization of states. For brevity, we omit the formal definitions for a rule to be $\mathbf{O S P} \mathbf{P}^{\mathcal{M}}$-implementable and $\mathbf{O S P}^{\mathcal{B}}$-implementable; these notions should be clear given this discussion and the analogous definition for pure strategies.

Altogether, we find that for deterministic rules, our revelation principle is robust to player randomization:

THEOREM 3: If a deterministic rule $f$ is either

(i) $\mathbf{O S P}^{\mathcal{S}}$-implementable,

(ii) $\operatorname{OSP}^{\mathcal{M}}$-implementable, or

(iii) $\operatorname{OSP}^{\mathcal{B}}$-implementable, 
then it has a round table implementation, which is an $\mathbf{O S P}^{\mathcal{C}}$-implementation.

The proof is in Appendix E. On the one hand, we find that any deterministic rule that can be implemented by taking advantage of agent randomization can also be implemented with each agent playing a pure strategy, which is obviously dominant to him even if he worries that the other agents might randomize. On the other hand, it is unclear that this is true for stochastic rules. Altogether, then, while it is possible to reassure agents that they need not randomize or worry about other agents randomizing, it is difficult to rely on the agents to provide the desired randomization themselves; this should be provided by the administrator.

\subsection{Justification for ordinal mechanisms}

In this section, we state a result inspired by a recent paper (Carroll, 2017), who seeks a justification for stochastic rules that elicit only ordinal information from the agents about their preferences over outcomes.

Suppose that we have a finite set of outcomes, and each agent's type space consists of von Neumann-Morgenstern utility functions representing preferences over lotteries. Moreover, each agent's type is compatible with a strict ranking over outcomes. Let us say that a stochastic rule is ordinal if for each $i \in N$, each $\theta_{-i} \in \Theta_{-i}$, and each pair $\theta_{i}, \theta_{i}^{\prime} \in \Theta_{i}$ compatible with the same ranking, $f\left(\theta_{i}, \theta_{-i}\right)=f\left(\theta_{i}^{\prime}, \theta_{-i}\right)$. In other words, a stochastic rule is ordinal if it only depends on the agents' rankings of deterministic outcomes. Finally, let a randomized ordinal round table implementation be a randomized round table implementation when each agent's type space is replaced by the associated space of rankings.

Theorem 4: Suppose there is a finite set of outcomes and each agent has a von NeumannMorgenstern type compatible with a strict ranking over outcomes. If a stochastic rule $f$ has a random $\mathbf{O S P}^{\mathcal{S}}$-implementation, then $f$ is ordinal and has a randomized ordinal round table implementation.

The proof is in Appendix F. In this sense, obvious strategy-proofness provides a foundation for ordinal mechanisms. This observation was inspired by Carroll (2017), who provides a foundation involving the interdependence of the agents' preferences over lotteries. The alternative foundation offered here does not rely on interdependence as it involves a stronger solution concept.

\subsection{Round table mechanisms and ordinary strategy-proofness}

It turns out to be fruitful to consider standard strategy-proofness in the context of round table mechanisms, a point I am grateful to Shengwu Li for suggesting to me. For brevity, we gather the necessary definitions, which were introduced in sequence for obvious strategy-proofness, here:

Definition: Strategy-proofness.

- A pure strategy $S_{i} \in \mathcal{S}_{i}$ is dominant if for each $S_{i}^{\prime} \in \mathcal{S}_{i}$, each $S_{-i} \in \mathcal{S}_{-i}$, and each $\omega_{H_{0}} \in \Omega_{H_{0}}$, 


$$
\begin{gathered}
x^{G}\left(h_{\emptyset}, S_{i}, S_{-i}, B_{0}\left(\omega_{H_{0}}\right)\right) \\
R_{i} \\
x^{G}\left(h_{\emptyset}, S_{i}^{\prime}, S_{-i}, B_{0}\left(\omega_{H_{0}}\right)\right) .
\end{gathered}
$$

- A pure strategy profile $S$ belongs to $\mathbf{S P}(G, \theta)$ if and only if for each agent $i, S_{i}$ is dominant.

- A deterministic rule $f$ is $\mathbf{S P}$-implementable if there are

(i) $G \in \Gamma$, and

(ii) an associated Harsanyi pure strategy profile $\mathbb{S}$,

such that for each $\theta \in \Theta$,

(i) $\left(\mathbb{S}_{i}\left(\theta_{i}\right)\right) \in \mathbf{S P}(G, \theta)$, and

(ii) for each $\omega_{H_{0}} \in \Omega_{H_{0}}$,

$$
x^{G}\left(h_{\emptyset},\left(\mathbb{S}_{i}\left(\theta_{i}\right)\right)_{i \in N}, B_{0}\left(\omega_{H_{0}}\right)\right)=f(\theta) .
$$

In this case we say $G$ SP-implements $f$ through $\mathbb{S}$.

Which mechanisms SP-implement a given rule? It could be that there are none, but if there are any, then by the standard revelation principle (Gibbard, 1973; Myerson, 1981), at least one of them is a direct mechanism. Beyond this, very little is known in general, but for our purposes, all that matters is whether or not one of them is a round table mechanism:

Theorem 5: A deterministic rule $f$ has an $\mathbf{O S P}^{\mathcal{C}}$-implementation if and only if it has a $\mathbf{S P}$-implementation through a round table mechanism.

See Figure 1; the proof is in Appendix G. Thus the analysis of whether a deterministic rule has an obviously strategy-proof implementation may be safely confined to a simple class of mechanisms and a familiar solution concept.

\section{Appendix A}

In this appendix, we prove Proposition 1. We begin by summarizing some known results about game trees. As we do not work directly with conditions in marked by quotation marks, and the reader is referred to the cited sources for their definitions. By contrast, we do work with the italicized conditions, which are defined after our summary.

We wish for our extensive game forms to be compatible with a large class of game trees, subject to the "admissibility" requirement that any choices determine a unique play. Fortunately, Alós-Ferrer and Ritzberger (2008) (henceforth, AFR) have provided (independent) necessary and sufficient conditions:

Theorem (AFR, Theorem 6): ${ }^{9}$ A rooted pseudotree is "admissible" if and only if it satisfies "weak up-discreteness," "coherence," and regularity.

${ }^{9}$ A correction to the definition of "admissible" is provided in Alós-Ferrer, Kern, and Ritzberger (2011), and the most complete treatment of this result is given in Alós-Ferrer and Ritzberger (2016). 

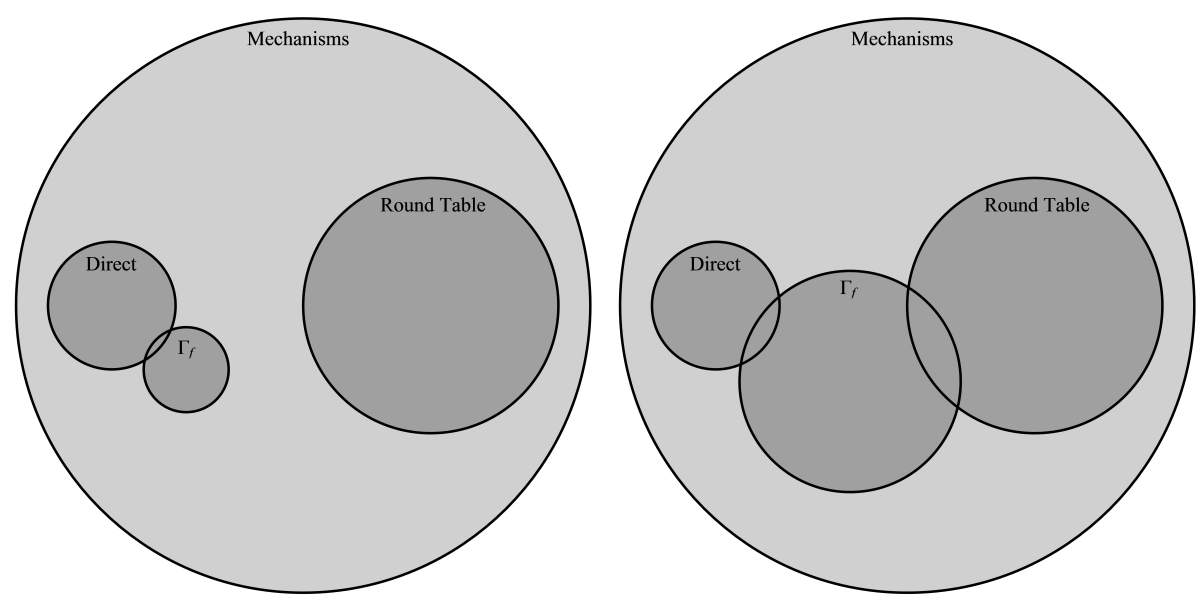

Figure 1: Theorem 5. For a given deterministic rule $f$, let $\Gamma_{f}$ be the class of game forms that SP-implement $f$. In general, $\Gamma_{f}$ may be empty (not pictured). Otherwise, by the classic revelation principle, $\Gamma_{f}$ intersects the class of direct mechanisms. If $\Gamma_{f}$ does not intersect the class of round table mechanisms (left), then $f$ has no OSP-implementation. On the other hand, if $\Gamma_{f}$ does intersect the class of round table mechanisms (right), then those mechanisms in the intersection are OSP-implementations.

AFR work with set-based rooted pseudotrees, where each history is the collection of plays that include it and precedence is set inclusion; this is without loss of generality in a precise sense (Alós-Ferrer and Ritzberger, 2005b). Because "weak up-discreteness" and "coherence" are together equivalent to up-discreteness (AFR, Corollary 3), "admissibility" is equivalent to up-discreteness and regularity (AFR, Corollary 5). Moreover, up-discreteness is equivalent to being a tree (Lemma AFR 1).

Furthermore, "admissibility" trivially implies "coherence" and regularity, which imply "selectiveness" (AFR, Proposition 6b). ${ }^{10}$ "Selectiveness" implies "well-meeting" (AFR, Footnote 27) ${ }^{11}$ which is equivalent to being a meet-semilattice (Alós-Ferrer and Ritzberger, 2005a). Additionally, "selectiveness" implies regularity (FR, Proposition 6a).

These existing results together imply a regular rooted tree is a meet-semilattice (Lemma AFR 2). To assure the reader that this is indeed true in general for ordertheoretic pseudotrees, we provide a direct proof. We then make two novel observations: a meet-semilattice pseudotree is regular (Lemma 1.1), and a meet-semilattice tree is rooted (Lemma 1.2). It is immediate from the lemmas that "admissibility" is equivalent to being a (necessarily rooted) meet-semilattice tree.

Throughout, we assume the Axiom of Choice, which is equivalent to the Hausdorff Maximality Principle, which is equivalent to Zorn's Lemma. The Axiom of Choice guarantees that strategies exist.

For each $h \in H$, let $\uparrow h \equiv\left\{h^{\prime} \in H \mid h^{\prime} \precsim h\right\}$ and $\uparrow h \equiv(\uparrow h \backslash\{h\})$ denote the weak and strict predecessors of $h$, respectively. We restate the definitions from the text using this notation:

Definition: A pseudotree (Koppelberg and Monk, 1991) is a partially ordered set $(H, \precsim)$ such that for each $h \in H, \uparrow h$ is a chain. It is rooted if there is $\min H$.

\footnotetext{
${ }^{10}$ In fact, they show that regularity and a condition weaker than "coherence" imply "selectiveness".

${ }^{11}$ In this article, earlier histories are smaller, while in AFR, earlier histories are greater; thus we use "well-meeting" in place of their "well-joining."
} 
Definition: A tree (see the Jech, 1971 survey) is a partially ordered set $(H, \precsim)$ such that for each $h \in H, \uparrow h$ is well-ordered.

Definition: A pseudotree $(H, \precsim)$ is up-discrete if each nonempty chain has a minimum.

The following observation appears in Alós-Ferrer and Ritzberger (2008); we provide the simple proof for completeness:

LEMma AFR 1: A pseudotree is a tree if and only if it is up-discrete.

Proof: We prove the two implications in sequence.

$[\Rightarrow]$ Let $H^{\prime}$ be a nonempty chain. Then there is $h \in H^{\prime}$. Since $(H, \precsim)$ is tree, there is $h_{m} \equiv \uparrow h$. Let $h^{\prime} \in H^{\prime}$. Since $H^{\prime}$ is a chain, either $h^{\prime} \precsim h$ or $h \prec h^{\prime}$. In the former case, $h^{\prime} \in \uparrow h$, so $h_{m} \precsim h^{\prime}$ by construction; in the latter case, $h_{m} \precsim h \prec h$. Since $h^{\prime} \in H^{\prime}$ was arbitrary, thus $h_{m}=\min H^{\prime}$.

$[\Leftarrow]$ Let $h \in H$ and let $H^{\prime} \subseteq \uparrow h, H^{\prime} \neq \emptyset$. Since $(H, \precsim)$ is a pseudotree, thus $\uparrow h$ is a chain, so $H^{\prime}$ is a nonempty chain. By up-discreteness, $H^{\prime}$ has a minimum. Since $H^{\prime}$ was arbitrary, thus $\uparrow h$ is well-ordered.

Before proceeding, we introduce some more notation. For each $H^{*} \subseteq H$, let $\mathcal{U}\left(H^{*}\right)$ denote the set of upper bounds of $H^{*}$. Recall that $\sup H^{*}=\min \mathcal{U}\left(H^{*}\right)$; since $\precsim$ is a partial order, if $\sup H^{*}$ exists then it is unique. Similarly, let $\mathcal{L}\left(H^{*}\right)$ denote the set of lower bounds of $H^{*}$, and recall that inf $H^{*}=\max \mathcal{L}\left(H^{*}\right)$, which is unique if it exists. Finally, we write $h \wedge h^{\prime}$ for the meet of $h$ and $h^{\prime}$, $\inf \left\{h, h^{\prime}\right\}$, which is unique if it exists.

Definition: A meet-semilattice is a partially ordered set $(H, \precsim)$ such that for each pair $h, h^{\prime} \in H$, there is $h \wedge h^{\prime}$.

Definition: A pseudotree $(H, \precsim)$ is regular if for each $h \in H$ such that $(\uparrow \uparrow h) \neq \emptyset$, there is $\sup (\uparrow \uparrow h)$.

We now prove the lemmas in sequence.

LEMmA AFR 2: If a rooted tree is regular, then it is a meet-semilattice.

Proof: Let $h, h^{\prime} \in H$. If $h$ and $h^{\prime}$ can be compared, then trivially there is $h \wedge h^{\prime} \in\left\{h, h^{\prime}\right\}$, so assume $h$ and $h^{\prime}$ cannot be compared.

Since $(H, \precsim)$ is a tree, both $\uparrow h$ and $\uparrow h^{\prime}$ are well-ordered. Since $h \in\left(\uparrow h \backslash \uparrow h^{\prime}\right)$ and $h^{\prime} \in\left(\uparrow h^{\prime} \backslash \uparrow h\right)$, thus there are $h_{m} \equiv \min \left(\uparrow h \backslash \uparrow h^{\prime}\right)$ and $h_{m}^{\prime} \equiv \min \left(\uparrow h^{\prime} \backslash \uparrow h\right)$. Notice that $h_{m}$ and $h_{m}^{\prime}$ cannot be compared: $h_{m} \precsim h_{m}^{\prime}$ implies $h_{m} \in \uparrow h^{\prime}$, while $h_{m}^{\prime} \precsim h_{m}$ implies $h_{m}^{\prime} \in \uparrow h$.

Define $H^{*} \equiv\left(\uparrow \uparrow h_{m}\right)$. Since $h_{m} \not h_{m}^{\prime}$, thus $h_{m}$ is not the root, so $H^{*}$ is not empty as it includes the root. We claim $H^{*}=\uparrow h \cap \uparrow h^{\prime}$.

To see $H^{*} \subseteq \uparrow h \cap \uparrow h^{\prime}$, let $h^{*} \in H^{*}$. Then $h^{*} \prec h_{m} \precsim h$, so $h^{*} \in \uparrow h$. Since $h^{*} \prec h_{m}=\min \left(\uparrow h \backslash \uparrow h^{\prime}\right)$, thus $h^{*} \in \uparrow h \cap \uparrow h^{\prime}$. 
Conversely, let $h^{*} \in \uparrow h \cap \uparrow h^{\prime}$. Then $h^{*} \notin\left\{h_{m}, h_{m}^{\prime}\right\}$. Since $\uparrow h$ and $\uparrow h^{\prime}$ are chains, thus $h^{*}$ can be compared to $h_{m}$ and $h^{*}$ can be compared to $h_{m}^{\prime}$. But we cannot have $h_{m} \prec h^{*}$, else $h_{m}$ and $h_{m}^{\prime}$ can be compared: if $h^{*} \prec h_{m}^{\prime}$, by transitivity; if $h_{m}^{\prime} \prec h^{*}$, because $\uparrow h^{*}$ is a chain as $(H, \precsim)$ is a tree. Thus $h^{*} \prec h_{m}$, so $h^{*} \in H^{*}$.

Altogether, $H^{*}=\uparrow h \cap \uparrow h^{\prime}$, as desired. We next claim $H^{*}$ has a maximum. Indeed, assume by way of contradiction that $H^{*}$ has no maximum. Since $H^{*}=\left(\uparrow \uparrow h_{m}\right)$ is nonempty, by regularity it has a supremum, so $\mathcal{U}\left(H^{*}\right)$ has a minimum. Since $h_{m} \in \mathcal{U}\left(H^{*}\right)$, and since $h^{*} \prec h_{m}$ implies $h^{*} \in H^{*}$ which implies $h^{*} \notin \mathcal{U}\left(H^{*}\right)$, thus $h_{m}=\min \mathcal{U}\left(H^{*}\right)$. But $H^{*} \subseteq \uparrow h^{\prime}$, so $h^{\prime} \in \mathcal{U}\left(H^{*}\right)$ and $h_{m} \not{L} h^{\prime}$, contradicting that $h_{m}=\min \mathcal{U}\left(H^{*}\right)$. Thus $H^{*}$ has maximum $h_{M}^{*}$, as desired.

Finally, we claim $h_{M}^{*}=h \wedge h^{\prime}$. Indeed, since $h_{M}^{*}=\max \uparrow h \cap \uparrow h^{\prime}$, thus $h_{M}^{*} \in \mathcal{L}\left(\left\{h, h^{\prime}\right\}\right)$. Assume, by way of contradiction, there is $h^{*} \in \mathcal{L}\left(\left\{h, h^{\prime}\right\}\right)$ such that $h_{M}^{*} \prec h^{*}$. Since $\uparrow h$ is a chain, thus $h^{*}$ and $h_{m}$ can be compared. Moreover, $h_{M}^{*}=\max \uparrow \uparrow h_{m}$ and $h_{M}^{*} \prec h^{*}$, so $h_{m} \precsim h^{*}$. But then $h_{m} \precsim h^{*} \precsim h^{\prime}$, so $h_{m} \in \uparrow h^{\prime}$, contradicting $h_{m}=\min \uparrow h \backslash \uparrow h^{\prime}$. Thus $h_{M}^{*}=\max \mathcal{L}\left(\left\{h, h^{\prime}\right\}\right)=h \wedge h^{\prime}$, as desired.

LEMmA 1.1: If a pseudotree is a meet-semilattice, then it is regular.

Proof: Assume, by way of contradiction, $(H, \precsim)$ is not regular. Then there is $h \in H$ such that $\uparrow \uparrow h$ is nonempty with no supremum.

Assume, by way of contradiction, $\uparrow \uparrow h \cap \mathcal{U}(\uparrow \uparrow h) \neq \emptyset$. Since $(H, \precsim)$ is a pseudotree, thus $\uparrow \uparrow h$ is chain. Then for each pair $h^{\prime}, h^{\prime \prime} \in \uparrow \uparrow h \cap \mathcal{U}(\uparrow \uparrow h)$, since $h^{\prime} \not h^{\prime \prime}$ and $h^{\prime \prime} \not h^{\prime}$, thus $h^{\prime} \sim h^{\prime \prime}$, so $h^{\prime}=h^{\prime \prime}$. But then $\uparrow \uparrow h \cap \mathcal{U}(\uparrow \uparrow h)$ is a singleton, so there is $\min \uparrow \uparrow h \cap \mathcal{U}(\uparrow \uparrow h)=$ $\max \uparrow \uparrow h=\sup \uparrow \uparrow h$, contradicting that there is no $\sup \uparrow \uparrow$. Thus $\uparrow \uparrow h \cap \mathcal{U}(\uparrow \uparrow h)=\emptyset$, as desired.

By construction, $h \in \mathcal{U}(\uparrow \uparrow h)$. Moreover, for each $h^{\prime} \in H, h^{\prime} \prec h$ implies $h^{\prime} \in \uparrow \uparrow h$, which implies $h^{\prime} \notin \mathcal{U}(\uparrow \uparrow h)$. Since $h \neq \min \mathcal{U}(\uparrow \uparrow h)$, and since $h^{\prime} \in \mathcal{U}(\uparrow \uparrow h)$ implies $h^{\prime} \nprec h$, thus there is $h^{\prime} \in \mathcal{U}(\uparrow \uparrow h)$ such that $h$ and $h^{\prime}$ cannot be compared.

Since $(H, \precsim)$ is a meet-semilattice, there is $h^{*} \equiv h \wedge h^{\prime}$. Since $h$ and $h^{\prime}$ cannot be compared, thus $h^{*} \notin\left\{h, h^{*}\right\}$, so $h^{*} \in \uparrow \uparrow h$. Since $\uparrow h$ is a chain with no maximum, there is $h^{* *} \in \uparrow \uparrow h$ such that $h^{*} \prec h^{* *}$. But then since $h, h^{\prime} \in \mathcal{U}(\uparrow \uparrow h)$, thus $h^{* *} \in \mathcal{L}\left(\left\{h, h^{\prime}\right\}\right)$ and $h^{*} \prec h^{* *}$, contradicting that $h^{*}=h \wedge h^{\prime}$. Thus $(H, \precsim)$ is regular, as desired.

LEMMA 1.2: Every meet-semilattice tree is rooted.

Proof: Let $(H, \precsim)$ satisfy the hypotheses. By Lemma AFR $1,(H, \precsim)$ is up-discrete, so each nonempty chain has a lower bound. Thus by Zorn's Lemma, $H$ has minimal element $h_{\emptyset}$.

Assume, by way of contradiction, $H$ has two distinct minimal elements $h_{\emptyset}$ and $h_{\emptyset}^{\prime}$. By meet-closure, there is $h^{*} \equiv h_{\emptyset} \wedge h_{\emptyset}^{\prime}$. Since $h_{\emptyset}$ and $h_{\emptyset}^{\prime}$ cannot be compared, thus $h^{*} \notin\left\{h_{\emptyset}, h_{\emptyset}^{\prime}\right\}$. But then $h^{*} \prec h_{\emptyset}$, contradicting the minimality of $h_{\emptyset}$. Thus $h_{\emptyset}=\min H$.

Altogether, we have established:

Proposition 1: The class of meet-semilattice trees is the class of "admissible" rooted pseudotrees. 


\section{Appendix B}

In this appendix, we prove Proposition 2. The overall approach is to take an arbitrary deterministic rule $f$ and assume that some game form $G \mathbf{O S P}^{\mathcal{S}}$-implements $f$ through some Harsanyi pure profile $\mathbb{S}$, then successively transform the pair $(G, \mathbb{S})$ until we have a round table implementation of $f$.

We first remove all of the administrator's moves; this involves 'pruning' off irrelevant branches as in Li (2016), as well as 'squishing' nodes that have a single immediate successor. Formally:

Definition: Tree operations. Let $(H, \precsim)$ be a meet-semilattice tree, let $H^{\prime} \subseteq H$ be nonempty, and let $\precsim$ be the restriction of $\precsim$ to $H^{\prime}$. Then

- $\left(H^{\prime}, \precsim^{\prime}\right)$ is a pruning of $(H, \precsim)$ if there is $E \subseteq H$ such that

$$
H^{\prime}=H \backslash\{h \in H \mid \text { there is } e \in E \text { such that } e \precsim h\} \text {, and }
$$

- $\left(H^{\prime}, \precsim\right)$ is a squishing of $(H, \precsim)$ if there is $H^{*} \subseteq H$ such that

(i) for each $h^{*} \in H^{*}, h^{*}$ has one immediate successor in $H$, and

(ii) $H^{\prime}=H \backslash H^{*}$.

Because we use a particularly large game trees, one might worry that these operations might result in an illegal partially ordered set. Fortunately, however, this is not the case.

First, we prove that pruning a meet-semilattice tree produces a meet-semilattice tree:

LEMmA 2.1: If $(H, \precsim)$ is a meet-semilattice tree and $\left(H^{\prime}, \precsim^{\prime}\right)$ is a pruning of $(H, \precsim)$, then $\left(H^{\prime}, \precsim^{\prime}\right)$ is a meet-semilattice tree.

Proof: Let $(H, \precsim)$ and $\left(H^{\prime}, \precsim \prime\right)$ be as in the hypothesis. Then there is $E \subseteq H$ such that $H^{\prime}=H \backslash\{h \in H \mid$ there is $e \in E$ such that $e \precsim h\}$.

We first claim that for each $h^{\prime} \in H^{\prime},\left\{h \in H^{\prime} \mid h \precsim h^{\prime}\right\}=\left\{h \in H \mid h \precsim h^{\prime}\right\}$. Indeed, let $h^{\prime} \in H^{\prime}$. By construction, $\left\{h \in H^{\prime} \mid h \precsim h^{\prime}\right\} \subseteq\left\{h \in H \mid h \precsim h^{\prime}\right\}$. Conversely, let $h \in H$ such that $h \precsim h^{\prime}$, and assume, by way of contradiction, $h \notin H^{\prime}$. Then there is $e \in E$ such that $e \precsim h$. But then by transitivity, $e \precsim h^{\prime}$, so $h^{\prime} \notin H^{\prime}$, contradicting $h^{\prime} \in H^{\prime}$. Altogether, $\left\{h \in H^{\prime} \mid h \precsim h^{\prime}\right\}=\left\{h \in H \mid h \precsim h^{\prime}\right\}$, as desired.

To see $\left(H^{\prime}, \precsim^{\prime}\right)$ is a tree, let $h^{\prime} \in H^{\prime}$. By the claim, $h^{\prime}$ has the same predecessors in $H$ and $H^{\prime}$. Moreover, this set is well-ordered since $(H, \precsim)$ is a tree. Since $h^{\prime} \in H^{\prime}$ was arbitrary, we are done.

To see $\left(H^{\prime}, \precsim^{\prime}\right)$ is a meet-semilattice, let $h, h^{\prime} \in H^{\prime}$. Since $h$ has the same predecessors in $H$ and $H^{\prime}$, and since $h^{\prime}$ does as well, thus $h$ and $h^{\prime}$ have the same lower bounds in $H$ and $H^{\prime}$. Moreover, this set has a maximum since $(H, \precsim)$ is a meet-semilattice. Since $h, h^{\prime} \in H^{\prime}$ were arbitrary, we are done.

Squishing a meet-semilattice tree produces a meet-semilattice tree, as well:

Lemma 2.2 : If $(H, \precsim)$ is a meet-semilattice tree and $\left(H^{\prime}, \precsim^{\prime}\right)$ is a squishing of $(H, \precsim)$, then $\left(H^{\prime}, \precsim \prime\right)$ is a meet-semilattice tree. 
Proof: Let $(H, \precsim)$ and $\left(H^{\prime}, \precsim^{\prime}\right)$ be as in the hypothesis. Then there is $H^{*} \subseteq H$ such that for each $h^{*} \in H^{*}, h^{*}$ has one immediate successor in $H$, and (ii) $H^{\prime}=H \backslash H^{*}$.

To see $\left(H^{\prime}, \precsim^{\prime}\right)$ is a tree, let $h^{\prime} \in H^{\prime}$ and let $H^{*} \subseteq\left\{h \in H^{\prime} \mid h \precsim h^{\prime}\right\}$. Then $H^{*} \subseteq\{h \in$ $\left.H \mid h \precsim h^{\prime}\right\}$. Since $(H, \precsim)$ is a tree, $\left\{h \in H \mid h \precsim h^{\prime}\right\}$ is well-ordered, so $H^{*}$ has a minimum. Since $H^{*} \subseteq\left\{h \in H^{\prime} \mid h \precsim h^{\prime}\right\}$ was arbitrary, thus $\left\{h \in H^{\prime} \mid h \precsim h^{\prime}\right\}$ is well-ordered. Since $h^{\prime} \in H^{\prime}$ was arbitrary, we are done.

Next, we claim that for each pair $h, h^{*} \in H, h^{*} \prec h$ implies $h^{*}$ has an immediate successor $h^{* *} \in H$ such that $h^{* *} \precsim h$. Indeed, let $h, h^{*} \in H$ with $h^{*} \prec h$. Since $(H, \precsim)$ is a tree and $\left\{h^{\prime} \in H \mid h^{*} \prec h^{\prime} \precsim h\right\}$ is a nonempty subset of $\left\{h^{\prime} \in H \mid h^{\prime} \precsim h\right\}$, thus $\left\{h^{\prime} \in H \mid h^{*} \prec h^{\prime} \precsim h\right\}$ has minimum $h^{* *}$. There cannot be $h^{* * *}$ such that $h^{*} \prec h^{* * *} \prec h^{* *}$, else we would have $h^{* * *} \in\left\{h^{\prime} \in H \mid h^{*} \prec h^{\prime} \precsim h\right\}$, contradicting the minimality of $h^{* *}$. Thus $h^{* *} \in H$ is an immediate successor of $h^{*} \in H$ such that $h^{* *} \precsim h$, as desired.

Assume, by way of contradiction, $\left(H^{\prime}, \precsim^{\prime}\right)$ is not a meet-semilattice. Then there are $h, h^{\prime} \in H^{\prime}$ with no meet in $H^{\prime}$. Since $(H, \precsim)$ is a meet-semilattice, $h$ and $h^{\prime}$ have meet $h^{*}$ in $H$. If $h^{*}$ were in $H^{\prime}$, then it would be the meet of $h$ and $h^{\prime}$ in $H^{\prime}$, which does not exist; thus $h^{*} \in H \backslash H^{\prime}$. In particular, $h^{*} \notin\left\{h, h^{\prime}\right\}$, so $h^{*} \prec h$ and $h^{*} \prec h^{\prime}$. By the claim, $h^{*}$ has immediate successors $h^{* *}$ and $h^{* * *}$ such that $h^{* *} \precsim h$ and $h^{* * *} \precsim h^{\prime}$. But $h^{*} \in H \backslash H^{\prime}$, so $h^{*}$ has a unique immediate successor, so $h^{* *}=h^{* * *}$, so $h^{* *}$ is a lower bound of $h$ and $h^{\prime}$ that is greater than $h^{*}$, contradicting that $h^{*}$ is the meet of $h$ and $h^{\prime}$.

At this point, we proceed to take pairs $(G, \mathbb{S})$ and transform them into pairs $(\mathcal{T}(G), \mathcal{T}(\mathbb{S}))$ according to a series of transformations $\mathcal{T}$ while preserving implementation. As promised, the first transformation removes the administrator from the game form:

Definition: Transformation $\mathcal{T}_{\omega_{H_{0}}}$.

- For each $\omega_{H_{0}} \in \Omega_{H_{0}}$, define $\mathcal{T}_{\omega_{H_{0}}}(G)$ by $G^{\prime}=\left\langle H^{\prime}, \precsim^{\prime}, A^{\prime}, \mathcal{A}^{\prime}, P^{\prime},\left(\mathcal{I}_{i}^{\prime}\right)_{i \in N}, g^{\prime}\right\rangle$ :

(i) $C_{0} \equiv B_{0}\left(\omega_{H_{0}}\right)$,

(ii) $E \equiv \cup_{h \in H_{0}}\left\{h^{\prime} \in \sigma(h) \mid \mathcal{A}\left(h^{\prime}\right) \neq C_{0}(h)\right\}$

(iii) $H^{*} \equiv H \backslash\{h \in H \mid$ there is $e \in E$ such that $e \precsim h\}$,

(iv) $H^{\prime} \equiv H^{*} \backslash H_{0}$, and

(v) everything else is the associated restriction.

- Given $\mathbb{S}$ for $G$, let $\mathcal{T}_{\omega_{H_{0}}}(\mathbb{S})$ be the associated restriction for $\mathcal{T}_{\omega_{H_{0}}}(G)$.

LEMma 2.3: If $G \in \Gamma \mathbf{O S P}^{\mathcal{S}}$-implements $f$ through $\mathbb{S}$, then for each $\omega_{H_{0}} \in \Omega_{H_{0}}, \mathcal{T}_{\omega_{H_{0}}}(G)$ OSP $^{\mathcal{S}}$-implements $f$ through $\mathcal{T}_{\omega_{H_{0}}}(\mathbb{S})$.

We omit the proof as the notation obscures the logic. For a fixed realization of the administrator's states, the transformation first removes all successors of any node that the administrator passes over, then squishes the administrator's nodes. This is a composition of squishing with pruning, so the resulting game tree is admissible. In the transformed game form, whenever an agent $i$ evaluates a given strategy $S_{i}$, there are fewer possibilities; thus the worst-case scenario for $S_{i}$ can only improve, while the best-case scenario across alternatives can only worsen. Because this is true across agents and type profiles, $\mathbf{O S P}^{\mathcal{S}}$ implementation is preserved.

With the administrator removed, we next remove absent-mindedness. For a given history $h$, let $\mathcal{E}(h)$ denote the earliest predecessor of $h$ that shares an information set 
with $h$, which is well-defined in a tree as the predecessors of $h$ are well-ordered. If $\mathcal{E}(h) \prec h$, let $\alpha(h)$ denote the unique action at $\mathcal{E}(h)$ that yields a history which precedes $h$.

\section{Definition: Transformation $\mathcal{T}_{i}$.}

- For each $i \in N$, define $\mathcal{T}_{i}(G)$ by $G^{\prime}=\left\langle H^{\prime}, \precsim^{\prime}, A^{\prime}, \mathcal{A}^{\prime}, P^{\prime},\left(\mathcal{I}_{i}^{\prime}\right)_{i \in N}, g^{\prime}\right\rangle$ :

(i) $H_{i}^{*} \equiv\left\{h \in H_{i} \mid \mathcal{E}(h) \prec h\right\}$,

(ii) $E \equiv \cup_{h \in H_{i}^{*}}\left\{h^{\prime} \in \sigma(h) \mid \mathcal{A}\left(h^{\prime}\right) \neq \alpha(h)\right\}$,

(iii) $H^{*} \equiv H \backslash\{h \in H \mid$ there is $e \in E$ such that $e \precsim h\}$,

(iv) $H^{* *} \equiv\left\{h \in H_{i}|| \sigma(h) \mid=1\right\}$,

(v) $H^{\prime} \equiv H^{*} \backslash H^{* *}$, and

(vi) everything else is the associated restriction.

- Given $\mathbb{S}$ for $G$, let $\mathcal{T}_{i}(\mathbb{S})$ be the associated restriction for $\mathcal{T}_{i}(G)$.

Lemma 2.4: If $G \in \Gamma \operatorname{OSP}^{\mathcal{S}}$-implements $f$ through $\mathbb{S}$, then for each $i \in N, \mathcal{T}_{i}(G)$ OSP $^{\mathcal{S}}$-implements $f$ through $\mathcal{T}_{i}(\mathbb{S})$.

Again, we omit the proof as the notation obscures the logic. Suppose agent $i$ has some history $h$ that is strictly preceded by other histories in its information set, the earliest of which is $\mathcal{E}(h)$. If $i$ has any pure strategies that can reach $h,{ }^{12}$ then all prescribe the action $\alpha(h)$ at $\mathcal{E}(h)$, and thus also prescribe $\alpha(h)$ at $h$. Any histories that follow from a choice other than $\alpha(h)$ at $h$, then, are impossible, and are pruned in the transformation. After the pruning, $h$ only has one remaining immediate successor, and at this point is squished. Thus the transformation is again a composition of a squishing and a pruning, and in the transformed game form, $i$ is not absent-minded. The rule's recommendation is still reached at each type profile, as only impossible plays have been removed. Moreover, $i$ evaluates each strategy at fewer information sets, and as in the previous transformation, for each agent there are fewer possibilities at each information set; altogether, $\mathbf{O S P}^{\mathcal{S}}$ implementation is again preserved. The composition of the $\mathcal{T}_{i}$ transformations across $N$ yields a game form with no absent-mindedness where each history has at least two immediate successors.

Let $\Gamma^{*} \subseteq \Gamma$ be the subclass of game forms without absent-mindedness. At this point, we remove imperfect information entirely, a possibility first observed by Ashlagi and Gonczarowski (2016):

Definition: Transformation $\mathcal{T}_{*}$.

- For each $i \in N$, define $\mathcal{T}_{i}(G)$ by $G^{\prime}=\left\langle H, \precsim, A, \mathcal{A}, P,\left(\mathcal{I}_{i}^{\prime}\right)_{i \in N}, g\right\rangle$ :

(i) for each $i \in N, \mathcal{I}_{i}^{\prime} \equiv\left\{\{h\} \mid h \in H_{i}\right\}$, and

(ii) everything else is the same.

- Given $\mathbb{S}$ for $G$, let $\mathcal{T}_{*}(\mathbb{S})$ be the associated restriction for $\mathcal{T}_{*}(G)$.

\footnotetext{
${ }^{12}$ It could, of course, be the case that $i$ cannot reach one of his histories $h$ with any pure strategy. For example, suppose all of $i$ 's histories share an information set; at each history, the available actions are Left, Center, and Right; and reaching $h$ requires a Left and then a Right.
} 
Lemma 2.5 (Ashlagi and Gonczarowski, 2016): If $G \in \Gamma^{*} \mathbf{O S P}^{\mathcal{S}}$-implements $f$ through $\mathbb{S}$, then $\mathcal{T}_{*}(G) \operatorname{OSP}^{\mathcal{S}}$-implements $f$ through $\mathcal{T}_{*}(\mathbb{S})$.

We omit this proof for the same reason we omitted the last two. Because there is no absent-mindedness, each history is a possibility compatible with some pure strategy profile. When information sets are split into singletons, then, each agent considers fewer possibilities at each information set; as with the previous transformations, this implies that $\mathbf{O S P} \mathbf{S}^{\mathcal{S}}$-implementation is preserved.

We remark that Lemma 2.5 would not hold if the original game form included absentmindedness, as in this case the splitting of information sets could create new possibilities for agents to consider:

EXAMPLE 2: There is one agent with one possible type, and there are two outcomes, $x$ and $x^{\prime}$. The agent prefers $x$, but the rule $f$ specifies that $x^{\prime}$ should be selected. The extensive game form $G$ is borrowed from the absent-minded driver game (Piccione and Rubinstein, 1997): the agent first selects whether to stay or exit; if he exits, the game ends; if he stays, then he selects a second time whether to stay or exit, and in either case the game ends. The agent is absent-minded: both histories share an information set. The outcome function assigns $x$ only to stay-exit, and assigns $x^{\prime}$ otherwise. Though $G$ $\mathbf{O S P}^{\mathcal{S}}$-implements $f$ through the pure exit strategy, $\mathcal{T}_{*}(G)$ does not $\mathbf{O S P}^{\mathcal{S}}$-implement $f$.

Thus the order in which these transformations are applied is important. That said, if they are applied in the order they are presented, the resulting game form is one of perfect information. At this point, we can apply a final transformation due to Li (2016) for game forms with perfect recall:

Definition: Transformation $\mathcal{T}_{\mathbb{S}}$.

- For each $\mathbb{S}$, define $\mathcal{T}_{\mathbb{S}}(G)$ by $G^{\prime}=\left\langle H^{\prime}, \precsim^{\prime}, A^{\prime}, \mathcal{A}^{\prime}, P^{\prime},\left(\mathcal{I}_{i}^{\prime}\right)_{i \in N}, g^{\prime}\right\rangle$ :

(i) $H^{\prime} \equiv\left\{h \in H \mid\right.$ there is $\theta \in \Theta$ such that $\left.h \in \pi^{G}\left(h_{\emptyset},\left(\mathbb{S}_{i}\left(\theta_{i}\right)\right)_{i \in N}\right)\right\}$, and

(ii) everything else is the associated restriction.

- Given $\mathbb{S}$ for $G$, let $\mathcal{T}_{\mathbb{S}}(\mathbb{S})$ be the associated restriction for $\mathcal{T}_{\mathbb{S}}(G)$.

TheOREM L (LI, 2016): If perfect recall $G \mathbf{O S P}^{\mathcal{S}}$-implements $f$ through $\mathbb{S}$, then $\mathcal{T}_{\mathbb{S}}(G)$ $\mathrm{OSP}^{\mathcal{S}}$-implements $f$ through $\mathcal{T}_{\mathbb{S}}(\mathbb{S})$.

This last result allows us to use $\mathbb{S}$ to prune off unused parts of the game tree, and therefore to label each action with the collection of types who take it. Altogether, we have established:

Proposition 2: If a deterministic rule has an $\mathbf{O S P}^{\mathcal{S}}$-implementation, then it has a round table implementation, which is an $\mathbf{O S P}^{\mathcal{C}}$-implementation.

\section{Appendix C}

In this appendix, we prove Theorem 1: 
THEOREM 1: If a stochastic rule has a random $\mathbf{O S P}^{\mathcal{S}}$-implementation, then it has a randomized round table implementation, which is a random $\mathbf{O S P}^{\mathcal{C}}$-implementation.

Proof: Suppose $(G, \mathcal{A}, \mu)$ random $\operatorname{OSP}^{\mathcal{S}}$-implements $f$ through $\mathbb{S}$.

Let $\omega_{H_{0}} \in \Omega_{H_{0}}$. As argued for Lemma 2.3, fixing this realization of the administrator's states, we may first remove all successors of any node that the administrator passes over, then squish the administrator's nodes, resulting in an admissible game tree and preserving random $\mathbf{O S P}^{\mathcal{S}}$-implementation. Unlike Lemma 2.3, however, the transformed game random $\mathbf{O S P}^{\mathcal{S}}$-implements a different rule: we previously had implementation of a stochastic rule, and now have implementation of a deterministic rule. Relabel each remaining history $h$ as $h^{\omega_{H_{0}}}$, let $\left(\mathbb{S}_{i}^{\omega_{H_{0}}}\right)_{i \in N}$ denote the associated restriction of $\mathbb{S}$, and let $G^{\omega_{H_{0}}}$ denote the transformed game form, which has no administrator moves and $\mathbf{O S P}^{\mathcal{S}}$ implements the deterministic rule $f^{\omega_{H_{0}}}$ given by:

$$
f^{\omega_{H_{0}}}(\theta)=x^{G}\left(h_{\emptyset},\left(\mathbb{S}_{i}\left(\theta_{i}\right)\right)_{i \in N}, B_{0}\left(\omega_{H_{0}}\right)\right) .
$$

Define $G^{+} \in \Gamma$ such that (i) at the initial history, the administrator selects from $\Omega_{H_{0}}$, (ii) each initial action $\omega_{H_{0}}$ is followed by the game form $G^{\omega_{H_{0}}}$, which has no administrator moves, and (iii) the administrator's strategy $B_{0}^{+}$is the identity. For each $i \in N$, each $\theta_{i} \in \Theta_{i}$ and each of $i$ 's histories $h^{\omega_{H_{0}}}$, define $\mathbb{S}_{i}^{+}\left(\theta_{i}\right)\left(h^{\omega_{H_{0}}}\right) \equiv \mathbb{S}_{i}^{\omega_{H_{0}}}\left(\theta_{i}\right)\left(h^{\omega_{H_{0}}}\right)$. Clearly, for each $\theta \in \Theta,\left(\mathbb{S}_{i}^{+}\left(\theta_{i}\right)\right) \in \mathbf{O S P}^{\mathcal{S}}\left(G^{+}, \theta\right)$, since at each information set, each agent compares the same possibilities that he did at one of the game forms $G^{\omega_{H_{0}}}$.

Consider $\left(G^{+}, \mathcal{A}, \mu\right) \in \Gamma^{M P}$. By construction, for each $\theta \in \Theta$ and each $X^{\prime} \in \mathcal{X}$, the administrator states that lead to $X^{\prime}$-outcomes in $G^{+}$are precisely those that label actions whose ensuing game forms lead to $X^{\prime}$-outcomes in $G^{+}$, which are precisely those that lead to $X^{\prime}$-outcomes in $G$ :

$$
\begin{array}{r}
\left\{\omega_{H_{0}} \in \Omega_{H_{0}} \mid x^{G^{+}}\left(h_{\emptyset}^{+},\left(\mathbb{S}_{i}^{+}\left(\theta_{i}\right)\right)_{i \in N}, B_{0}^{+}\left(\omega_{H_{0}}\right)\right) \in X^{\prime}\right\} \\
=\left\{\omega_{H_{0}} \in \Omega_{H_{0}} \mid x^{G_{\omega_{0}}}\left(h_{\emptyset}^{\omega_{H_{0}}},\left(\mathbb{S}_{i}^{\omega_{H_{0}}}\left(\theta_{i}\right)\right)_{i \in N}\right) \in X^{\prime}\right\} \\
=\left\{\omega_{H_{0}} \in \Omega_{H_{0}} \mid x^{G}\left(h_{\emptyset},\left(\mathbb{S}_{i}\left(\theta_{i}\right)\right)_{i \in N}, B_{0}\left(\omega_{H_{0}}\right)\right) \in X^{\prime}\right\} .
\end{array}
$$

Because $\mu$ assigns the same number to the former in $\left(G^{+}, \mathcal{A}, \mu\right)$ as to the latter in $(G, \mathcal{A}, \mu)$, thus

$$
\mu\left(\left\{\omega_{H_{0}} \in \Omega_{H_{0}} \mid x^{G^{+}}\left(h_{\emptyset}^{+},\left(\mathbb{S}_{i}^{+}\left(\theta_{i}\right)\right)_{i \in N}, B_{0}^{+}\left(\omega_{H_{0}}\right)\right) \in X^{\prime}\right\}\right)=f(\theta)\left(X^{\prime}\right),
$$

so $\left(G^{+}, \mathcal{A}, \mu\right)$ random $\mathbf{O S P}^{\mathcal{S}}$-implements $f$.

It remains to transform $\left(G^{+}, \mathcal{A}, \mu\right)$ into a randomized round table mechanism $\left(G^{*}, \mathcal{A}, \mu\right)$ while preserving random $\mathbf{O S P}^{\mathcal{S}}$-implementation of $f$. To do so, simply use Proposition 2 to replace each game form $G^{\omega_{H_{0}}}$ with a round table mechanism that $\mathbf{O S P}^{\mathcal{S}}$-implements the same deterministic rule $f^{\omega_{H_{0}}}$, and let $\left(\mathbb{S}_{i}^{*}\right)_{i \in N}$ gather the associated strategies. By construction,

$$
\begin{array}{r}
\left\{\omega_{H_{0}} \in \Omega_{H_{0}} \mid x^{G^{*}}\left(h_{\emptyset}^{*},\left(\mathbb{S}_{i}^{*}\left(\theta_{i}\right)\right)_{i \in N}, B_{0}^{*}\left(\omega_{H_{0}}\right)\right) \in X^{\prime}\right\} \\
=\left\{\omega_{H_{0}} \in \Omega_{H_{0}} \mid x^{G^{+}}\left(h_{\emptyset}^{+},\left(\mathbb{S}_{i}^{+}\left(\theta_{i}\right)\right)_{i \in N}, B_{0}^{+}\left(\omega_{H_{0}}\right)\right) \in X^{\prime}\right\},
\end{array}
$$

and $\mu$ assigns the same number to the former in $\left(G^{*}, \mathcal{A}, \mu\right)$ as to the latter in $\left(G^{+}, \mathcal{A}, \mu\right)$, so $\left(G^{*}, \mathcal{A}, \mu\right)$ random $\mathbf{O S P}^{\mathcal{S}}$-implements $f$, as desired. That $\left(G^{*}, \mathcal{A}, \mu\right)$ is a randomized round table mechanism is obvious. 


\section{Appendix D}

In this appendix, we prove Theorem 2 :

TheOREM 2: Random serial dictatorship has no round table implementation.

ProOF: Let $f$ denote random serial dictatorship. Assume, by way of contradiction, that there is round table mechanism $G$ that $\mathbf{O S P}^{\mathcal{S}}$-implements $f$.

Let us say that a history is nondegenerate if it has more than one action. ${ }^{13}$ As $G$ $\operatorname{OSP}^{\mathcal{S}}$-implements $f$, there must be a sharp history $h$. As the game tree is a tree, the predecessors of $h$ are well-ordered, and thus $h$ has an earliest sharp predecessor $h^{*}$. As $G$ is a round table mechanism, $h^{*}$ is controlled by some agent $i \in N$ and the actions at $h^{*}$ are labeled by sets of types; as $h^{*}$ has no sharp predecessors, these actions partition $\Theta_{i}$.

Define $\theta_{i}^{*}$ by:

$$
\theta_{i}^{*}(a) \equiv\left\{\begin{array}{cc}
9, & a=A, \\
6, & a=B, \\
0, & a=C .
\end{array}\right.
$$

Let $\Theta_{*}$ be the action at $h^{*}$ that includes $\theta_{i}^{*}$, and let $\Theta^{\prime}$ be a distinct action at $h^{*}$.

Let us evaluate $\mathbb{S}_{i}\left(\theta_{i}^{*}\right)$ at $h^{*}$, supposing that $i$ has type $\theta_{i}^{*}$. As $h^{*}$ has no sharp predecessors and $G \mathbf{O S P}^{\mathcal{S}}$-implements $f$, thus the worst-case scenario from continuing with $\mathbb{S}_{i}\left(\theta_{i}^{*}\right)$ is an outcome where $i$ receives a uniform lottery over the three objects. Thus $\Theta^{\prime}$ cannot include a type where $A$ is top-ranked, else by similar logic the best-case scenario from deviating to $\Theta^{\prime}$ would be an outcome where $i$ receives $A$ with probability 1 , contradicting that $\mathbb{S}_{i}\left(\theta_{i}^{*}\right)$ is obviously dominant for type $\theta_{i}^{*}$. Moreover, $\Theta^{\prime}$ cannot include a type where $B$ is top-ranked, else by deviating it would be possible for $i$ to receive $B$ with probability 1 , contradicting that $\mathbb{S}_{i}\left(\theta_{i}^{*}\right)$ is obviously dominant for type $\theta_{i}^{*}$. As $\Theta^{\prime}$ was arbitrary, thus all actions at $h^{*}$ except for $\Theta_{*}$ only include types where $C$ is top-ranked.

Define $\theta_{i}^{* *}$ by:

$$
\theta_{i}^{* *}(a) \equiv \begin{cases}9, & a=A, \\ 6, & a=C, \\ 0, & a=B .\end{cases}
$$

Let us evaluate $\mathbb{S}_{i}\left(\theta_{i}^{* *}\right)$ at $h^{*}$, supposing that $i$ has type $\theta_{i}^{* *}$. Since $\theta_{i}^{* *}$ does not top-rank $C$, thus $\theta_{i}^{* *} \in \Theta_{*}$. Using the logic from the previous paragraph, the worst-case scenario from continuing with $\mathbb{S}_{i}\left(\theta_{i}^{* *}\right)$ is an outcome where $i$ receives a uniform lottery over the three objects, and by deviating to $\Theta^{\prime}$ (where necessarily all types top-rank $C$ ) it would be possible for $i$ to receive $C$ with probability 1 . But then $\mathbb{S}_{i}\left(\theta_{i}^{* *}\right)$ is not obviously dominant for type $\theta_{i}^{* *}$, contradicting that $G \mathbf{O S P}^{\mathcal{S}}$-implements $f$.

\section{Appendix E}

In this appendix, we prove Theorem 3.

We begin with two similar observations. First, if a deterministic rule has an $\mathbf{O S P}^{\mathcal{M}_{-}}$ implementation, then it has one in pure strategies:

\footnotetext{
${ }^{13}$ Bade and Gonczarowski (2017) observe that attention can be restricted to game trees for which all histories are nondegenerate.
} 
LEMMA 3.1: If $G \in \Gamma \mathbf{O S P}^{\mathcal{M}}$-implements $f$ through $\mathbb{M}$, then for each $\omega_{N} \in \Omega_{N}$, $G \operatorname{OSP}^{\mathcal{S}}$-implements $f$ through $\mathbb{S}$, where for each $i \in N$ and each $\theta_{i} \in \Theta_{i}, \mathbb{S}_{i}\left(\theta_{i}\right)=$ $\mathbb{M}_{i}\left(\theta_{i}\right)\left(\omega_{i}\right)$.

Proof: Suppose $G$ OSP $^{\mathcal{M}}$-implements $f$ through $\mathbb{M}$, and let $\omega_{N} \in \Omega_{N}$. For each $i \in N$ and each $\theta_{i} \in \Theta_{i}$, define $\mathbb{S}_{i}\left(\theta_{i}\right) \equiv \mathbb{M}\left(\theta_{i}\right)\left(\omega_{i}\right)$.

Let $\theta \in \Theta$ and let $i \in N$. Define $M_{i} \equiv \mathbb{M}_{i}\left(\theta_{i}\right)$ and define $S_{i} \equiv \mathbb{S}_{i}\left(\theta_{i}\right)$. We claim $S_{i}$ is obviously $\mathcal{S}$-dominant. Indeed, let $I_{i} \in \mathcal{I}_{i}\left(S_{i}\right)$. Since $S_{i}=M_{i}\left(\omega_{i}\right)$, thus $I_{i} \in \mathcal{I}_{i}\left(M_{i}\left(\omega_{i}\right)\right)$. Since $M_{i}$ is obviously dominant, for each pair $h, h^{\prime} \in I_{i}$, each $M_{i}^{\prime} \in \mathcal{M}_{i}$ such that $M_{i}\left(\omega_{i}\right)\left(I_{i}\right) \neq M_{i}^{\prime}\left(\omega_{i}\right)\left(I_{i}\right)$, each pair $M_{-i}, M_{-i}^{\prime} \in \mathcal{M}_{-i}$, each pair $\omega_{-i}, \omega_{-i}^{\prime} \in \Omega_{-i}$, and each pair $\omega_{H_{0}}, \omega_{H_{0}}^{\prime} \in \Omega_{H_{0}}$,

$$
\begin{gathered}
x^{G}\left(h, M_{i}\left(\omega_{i}\right),\left(M_{j}\left(\omega_{j}\right)\right)_{j \in N \backslash\{i\}}, B_{0}\left(\omega_{H_{0}}\right)\right) \\
R_{i} \\
x^{G}\left(h^{\prime}, M_{i}^{\prime}\left(\omega_{i}\right),\left(M_{j}^{\prime}\left(\omega_{j}^{\prime}\right)\right)_{j \in N \backslash\{i\}}, B_{0}\left(\omega_{H_{0}}^{\prime}\right)\right) .
\end{gathered}
$$

Moreover, $S_{i}=M_{i}\left(\omega_{i}\right)$, and each pure strategy is a realization of some mixed strategy. Thus for each pair $h, h^{\prime} \in I_{i}$, each $S_{i}^{\prime} \in \mathcal{S}_{i}$ such that $S_{i}\left(I_{i}\right) \neq S_{i}^{\prime}\left(I_{i}\right)$, each pair $S_{-i}, S_{-i}^{\prime} \in$ $\mathcal{S}_{-i}$, and each pair $\omega_{H_{0}}, \omega_{H_{0}}^{\prime} \in \Omega_{H_{0}}$,

$$
\begin{gathered}
x^{G}\left(h, S_{i}, S_{-i}, B_{0}\left(\omega_{H_{0}}\right)\right) \\
R_{i} \\
x^{G}\left(h^{\prime}, S_{i}^{\prime}, S_{-i}^{\prime}, B_{0}\left(\omega_{H_{0}}^{\prime}\right)\right),
\end{gathered}
$$

so $S_{i}$ is obviously dominant. Since $i \in N$ was arbitrary, thus $\left(\mathbb{S}_{i}\left(\theta_{i}\right)\right) \in \operatorname{OSP}^{\mathcal{S}}(G, \theta)$. Since $\theta \in \Theta$ was arbitrary, thus $G \mathbf{O S P}^{\mathcal{S}}$-implements $f$ through $\mathbb{S}$. Since $\left(\omega_{i}\right) \in \times \Omega_{i}$ was arbitrary, we are done.

Second, if a deterministic rule has an $\mathbf{O S P}^{\mathcal{B}}$-implementation, then it has one in pure strategies:

Lemma 3.2: If $G \in \Gamma \mathbf{O S P}^{\mathcal{B}}$-implements $f$ through $\mathbb{B}$, then for each $\omega_{H_{N}} \in \Omega_{H_{N}}$ such that for each pair $h$ and $h^{\prime}$ that share an information set, $\omega_{h}=\omega_{h^{\prime}}, G$ OSP $^{\mathcal{S}}$-implements $f$ through $\mathbb{S}$, where for each $i \in N$ and each $\theta_{i} \in \Theta_{i}, \mathbb{S}_{i}\left(\theta_{i}\right)=\mathbb{B}_{i}\left(\theta_{i}\right)\left(\omega_{H_{i}}\right)$.

Proof: Suppose $G$ OSP $^{\mathcal{B}}$-implements $f$ through $\mathbb{B}$, and let $\omega_{H_{N}} \in \times \Omega_{H_{N}}$ such that for each pair $h$ and $h^{\prime}$ that share an information set, $\omega_{h}=\omega_{h^{\prime}}$. For each $i \in N$ and each $\theta_{i} \in \Theta_{i}$, define $\mathbb{S}_{i}\left(\theta_{i}\right) \equiv \mathbb{B}\left(\theta_{i}\right)\left(\omega_{H_{i}}\right)$.

First, we claim that $\mathbb{S}$ is a Harsanyi pure strategy profile. Indeed, let $i \in N$, let $\theta_{i} \in \Theta_{i}$, let $I_{i} \in \mathcal{I}_{i}$, and let $h, h^{\prime} \in I_{i}$. Since $\mathbb{B}_{i}\left(\theta_{i}\right)$ is a behavioral strategy, it includes the same mapping at $h$ and $h^{\prime}$; since by hypothesis $\omega_{h}=\omega_{h^{\prime}}$, thus $\mathbb{B}_{i}\left(\theta_{i}\right)\left(\omega_{H_{i}}\right)\left(h_{i}\right)=\mathbb{B}_{i}\left(\theta_{i}\right)\left(\omega_{H_{i}}\right)\left(h_{i}^{\prime}\right)$. Since $I_{i} \in \mathcal{I}_{i}$ and $h, h^{\prime} \in I_{i}$ were arbitrary, thus the choice function $\mathbb{B}_{i}\left(\theta_{i}\right)\left(\omega_{H_{i}}\right)$ is a pure strategy. Since $\theta_{i} \in \Theta_{i}$ was arbitrary, thus $\mathbb{S}_{i}$ is a Harsanyi pure strategy. Since $i \in N$ was arbitrary, thus $\mathbb{S}$ is a Harsanyi pure strategy profile, as desired.

Let $\theta \in \Theta$ and let $i \in N$. Define $B_{i} \equiv \mathbb{B}_{i}\left(\theta_{i}\right)$ and define $S_{i} \equiv \mathbb{S}_{i}\left(\theta_{i}\right)$. We claim $S_{i}$ is obviously $\mathcal{S}$-dominant. Indeed, let $I_{i} \in \mathcal{I}_{i}\left(S_{i}\right)$. By construction, $\mathcal{I}_{i}\left(S_{i}\right) \subseteq \mathcal{I}_{i}\left(B_{i}\right)$, so $I_{i} \in$ $\mathcal{I}_{i}\left(B_{i}\right)$. Since $B_{i}$ is obviously dominant, for each pair $h, h^{\prime} \in I_{i}$, each $\omega_{x} \in \Omega_{h}=\Omega_{h^{\prime}}$, each $B_{i}^{\prime} \in \mathcal{B}_{i}$ such that $B_{i}\left(\omega_{x}\right) \neq B_{i}^{\prime}\left(\omega_{x}\right)$, each $\omega_{H_{i} \backslash\{h\}} \in \Omega_{H_{i} \backslash\{h\}}$, each $\omega_{H_{i} \backslash\{h\}}^{\prime} \in \Omega_{H_{i} \backslash\{h\}}$, each pair $B_{-i}, B_{-i}^{\prime} \in \mathcal{B}_{-i}$, each pair $\left(\omega_{H_{j}}^{*}\right)_{j \in N \backslash\{i\}},\left(\omega_{H_{j}}^{\prime}\right) \in \times_{N \backslash\{i\}} \Omega_{H_{j}}$, and each pair $\omega_{H_{0}}, \omega_{H_{0}}^{\prime} \in$ $\Omega_{H_{0}}$ 


$$
\begin{gathered}
x^{G}\left(h, B_{i}\left(\omega_{x}, \omega_{H_{i} \backslash\{h\}}^{*}\right),\left(B_{j}\left(\omega_{H_{j}}\right)\right)_{j \in N \backslash\{i\}}, B_{0}\left(\omega_{H_{0}}\right)\right) \\
R_{i} \\
x^{G}\left(h^{\prime}, B_{i}^{\prime}\left(\omega_{x}, \omega_{H_{i} \backslash\left\{h^{\prime}\right\}}^{\prime}\right),\left(B_{j}^{\prime}\left(\omega_{H_{j}}^{\prime}\right)\right)_{j \in N \backslash\{i\}}, B_{0}\left(\omega_{H_{0}}^{\prime}\right)\right) .
\end{gathered}
$$

In particular, this holds for $\left(\omega_{x}, \omega_{H_{i} \backslash\{h\}}^{*}\right)=\omega_{H_{i}}$. Moreover, each pure strategy is a realization of some behavioral strategy. Thus for each pair $h, h^{\prime} \in I_{i}$, each $S_{i}^{\prime} \in \mathcal{S}_{i}$ such that $S_{i}\left(I_{i}\right) \neq S_{i}^{\prime}\left(I_{i}\right)$, each pair $S_{-i}, S_{-i}^{\prime} \in \mathcal{S}_{-i}$, and each pair $\omega_{H_{0}}, \omega_{H_{0}}^{\prime} \in \Omega_{H_{0}}$,

$$
\begin{gathered}
x^{G}\left(h, S_{i}, S_{-i}, B_{0}\left(\omega_{H_{0}}\right)\right) \\
R_{i} \\
x^{G}\left(h^{\prime}, S_{i}^{\prime}, S_{-i}^{\prime}, B_{0}\left(\omega_{H_{0}}^{\prime}\right)\right),
\end{gathered}
$$

so $S_{i}$ is obviously dominant. Since $i \in N$ was arbitrary, thus $\left(\mathbb{S}_{i}\left(\theta_{i}\right)\right) \in \operatorname{OSP}^{\mathcal{S}}(G, \theta)$. Since $\theta \in \Theta$ was arbitrary, thus $G \mathbf{O S P}^{\mathcal{S}}$-implements $f$ through $\mathbb{S}$. Since $\left(\omega_{H_{N}}\right) \in \times \Omega_{H_{N}}$ satisfying the given restriction was arbitrary, we are done.

By Lemma 3.1, Lemma 3.2, and Proposition 2, if a rule has any kind of obviously strategy-proof implementation, then it has a round table implementation. Altogether, we have established:

THEOREM 3: If a deterministic rule $f$ is either

(i) $\mathbf{O S P}^{\mathcal{S}}$-implementable,

(ii) $\operatorname{OSP}^{\mathcal{M}}$-implementable, or

(iii) $\mathbf{O S P}^{\mathcal{B}}$-implementable,

then it has a round table implementation, which is an $\mathbf{O S P}^{\mathcal{C}}$-implementation.

\section{Appendix F}

In this appendix, we prove Theorem 4:

Theorem 4: Suppose there is a finite set of outcomes and each agent has a von NeumannMorgenstern type compatible with a strict ranking over outcomes. If a stochastic rule $f$ has a random $\mathbf{O S P}^{\mathcal{S}}$-implementation, then $f$ is ordinal and has a randomized ordinal round table implementation.

Proof: Suppose $f$ has a random $\mathbf{O S P}^{\mathcal{S}}$-implementation. Then by Theorem 1 , it has a random round table implementation.

Let $i \in N$, and let $\theta_{i}, \theta_{i}^{\prime} \in \Theta_{i}$ be a pair of von Neumann-Morgenstern utility functions that are compatible with the same ordinal preferences over outcomes. Let $H_{\theta_{i}, \theta_{i}^{\prime}}$ be the collection of histories where $i$ has one action that includes $\theta_{i}$ and another action that includes $\theta_{i}^{\prime}$. At each $h \in H_{\theta_{i}, \theta_{i}^{\prime}}$, let $X_{\theta_{i}}^{h}$ be the collection of outcomes that could occur when the strategy of reporting $\theta_{i}$ is followed from $h$, and let $X_{\theta_{i}^{\prime}}^{h}$ be the collection of outcomes that could occur when the strategy of reporting $\theta_{i}^{\prime}$ is followed from $h$. As truthful reporting of $\theta_{i}$ and truthful reporting of $\theta_{i}^{\prime}$ are both obviously dominant for $i$, and as both of these types share a strict ranking of outcomes, it follows that $X_{\theta_{i}}^{h}$ and $X_{\theta_{i}^{\prime}}^{h}$ are the same singleton.

Let $\theta_{-i} \in \Theta_{-i}$. By the above observation, the distribution over outcomes generated by administrator randomization for reports of $\left(\theta_{i}, \theta_{-i}\right)$ is the same as the distribution 
generated for $\left(\theta_{i}^{\prime}, \theta_{-i}\right)$. As we have a random round table implementation of $f$, thus $f\left(\theta_{i}, \theta_{-i}\right)=f\left(\theta_{i}^{\prime}, \theta_{-i}\right)$; it follows that $f$ is ordinal.

Let $\left[\theta_{i}\right] \subseteq \Theta_{i}$ be the collection of types for $i$ that are compatible with the same ranking of outcomes as $\theta_{i}$. For each action that includes $\theta_{i}$, relabel the action to be the union of its previous types with the types in $\left[\theta_{i}\right]$. For each action that does not include $\theta_{i}$, relabel the action to be its previous types except for those in $\left[\theta_{i}\right]$. Finally, prune off all histories that follow from an action whose label is now empty. For each $\theta_{i}^{*} \in \Theta_{i}$ and each $\theta_{-i} \in \Theta_{-i}$, the reports $\left(\theta_{i}^{*}, \theta_{-i}\right)$ determine the same outcome for each round table mechanism selected by the administrator before and after this operation; thus the distribution generated by administrator randomization for reports $\left(\theta_{i}^{*}, \theta_{-i}\right)$ is the same before and after this operation, so we have a new round table implementation of $f$.

This relabeling and pruning can be done for each of the finitely many rankings for $i$, and then all of these stages of relabeling and pruning can be done for each of the finitely many agents. Finally, relabel each action by its associated rankings of its types; the result is clearly a random ordinal round table implementation of $f$.

\section{Appendix G}

In this appendix, we prove Theorem 5:

Theorem 5: A deterministic rule $f$ has an $\mathbf{O S P}^{\mathcal{C}}$-implementation if and only if it has a SP-implementation through a round table mechanism.

Proof: We prove the two implications in sequence. Note that because for each round table mechanism $G \in \Gamma^{R T}$, the administrator does not play $\left(H_{0}=\emptyset\right)$, we will suppress notation involving him.

$[\Rightarrow]$ Assume $f$ has an $\mathbf{O S P}^{\mathcal{C}}$-implementation. By Proposition 2, there are $G \in \Gamma^{R T}$ and an associated Harsanyi pure strategy profile $\mathbb{S}$ such that $G \mathbf{O S P}^{\mathcal{C}}$-implements $f$ through $\mathbb{S}$. For each $\theta \in \Theta$, since

- by definition, $\left(\mathbb{S}_{i}\left(\theta_{i}\right)\right) \in \operatorname{OSP}^{\mathcal{C}}(G, \theta)$;

- because $G \in \Gamma^{R T}, \operatorname{OSP}^{\mathcal{C}}(G, \theta)=\operatorname{OSP}^{\mathcal{S}}(G, \theta)$; and

- by definition, $\operatorname{OSP}^{\mathcal{S}}(G, \theta) \subseteq \mathbf{S P}(G, \theta)$;

thus altogether $\left(\mathbb{S}_{i}\left(\theta_{i}\right)\right) \in \mathbf{S P}(G, \theta)$. Since for each $\theta \in \Theta, x^{G}\left(h_{\emptyset},\left(\mathbb{S}_{i}\left(\theta_{i}\right)\right)_{i \in N}\right)=f(\theta)$, thus $G \mathbf{S P}$-implements $f$ through $\mathbb{S}$, as desired.

$[\Leftarrow]$ Assume there are $G \in \Gamma^{R T}$ and an associated Harsanyi pure strategy profile $\mathbb{S}$ such that $G \mathbf{S P}$-implements $f$ through $\mathbb{S}$. We claim that $G$ OSP $^{\mathcal{C}}$-implements $f$ through $\mathbb{S}$. Indeed, let $\theta \in \Theta$, let $i \in N$, and define $S_{i} \equiv \mathbb{S}_{i}\left(\theta_{i}\right)$.

We claim $S_{i}$ is obviously dominant. Indeed, let $I_{i} \in \mathcal{I}_{i}\left(S_{i}\right)$, let $h, h^{\prime} \in \mathcal{I}_{i}$, let $C_{i}^{\prime} \in \mathcal{C}_{i}$ such that $S_{i}\left(I_{i}\right) \neq C_{i}^{\prime}\left(I_{i}\right)$, and let $C_{-i}, C_{-i}^{\prime} \in \mathcal{C}_{-i}$. (As $G \in \Gamma^{R T}$, the administrator does not play; $\mathcal{C}_{0}=\emptyset$.)

Since $G \in \Gamma^{R T}, h=h^{\prime}$. Define $h_{1}$ to be the unique next history after $h$ determined by the action $S_{i}(h)$, and define $h_{2}$ to be the unique next history after $h$ determined by the action $C_{i}^{\prime}(h)$. Partition $H_{-i}$ into $H_{0}, H_{1}, H_{2}$, and $H_{*}$, where: 
- $H_{0} \equiv\left\{h^{\prime} \in H_{-i} \mid h^{\prime} \prec h\right\}$,

- $H_{1} \equiv\left\{h^{\prime} \in H_{-i} \mid h_{1} \precsim h^{\prime}\right\}$,

- $H_{2} \equiv\left\{h^{\prime} \in H_{-i} \mid h_{2} \precsim h^{\prime}\right\}$, and

- $H_{*} \equiv H_{-i} \backslash\left(H_{1} \cup H_{2}\right)$.

We use these to define $S_{-i} \in S_{-i}$. Since $G \in \Gamma^{R T}$, we can do so history-by-history:

- for each $h^{\prime} \in H_{0}$, define $j \equiv P\left(h^{\prime}\right)$, and define $S_{j}\left(h^{\prime}\right)$ to be the action that determines the unique next history in the (well-ordered) chain $\left\{h^{\prime \prime} \in H \mid h^{\prime \prime} \precsim h\right\}$;

- for each $h^{\prime} \in H_{1}$, define $j \equiv P\left(h^{\prime}\right)$, and define $S_{j}\left(h^{\prime}\right) \equiv C_{j}\left(h^{\prime}\right)$;

- for each $h^{\prime} \in H_{2}$, define $j \equiv P\left(h^{\prime}\right)$, and define $S_{j}\left(h^{\prime}\right) \equiv C_{j}^{\prime}\left(h^{\prime}\right)$; and

- for each $h^{\prime} \in H_{*}$, define $j \equiv P\left(h^{\prime}\right)$, and let $S_{j}\left(h^{\prime}\right) \in A\left(h^{\prime}\right)$ be arbitrary.

Define $S_{i}^{\prime} \in \mathcal{S}_{i}$ such that

- for each $h^{\prime} \in H_{i}$ such that $h^{\prime} \prec h, S_{i}^{\prime}\left(h^{\prime}\right)=S_{i}(h)$;

- for each $h^{\prime} \in H_{i}$ such that $h \precsim h^{\prime}, S_{i}^{\prime}\left(h^{\prime}\right)=C_{i}^{\prime}\left(h^{\prime}\right)$; and

- for all other $h^{\prime}, S_{i}^{\prime}\left(h^{\prime}\right) \in A\left(h^{\prime}\right)$ is arbitrary.

Since $S_{i}$ is dominant, we have

$$
\begin{gathered}
x^{G}\left(h_{\emptyset}, S_{i}, S_{-i}\right) \\
R_{i} \\
x^{G}\left(h_{\emptyset}, S_{i}^{\prime}, S_{-i}\right) .
\end{gathered}
$$

Since $\{h\}=I_{i} \in \mathcal{I}_{i}\left(S_{i}\right)$, by construction of $S_{-i}, h \in \pi^{G}\left(h_{\emptyset}, S_{i}, S_{-i}\right)$. Since $S_{i}$ and $S_{i}^{\prime}$ agree on histories that precede $h$, thus $h \in \pi^{G}\left(h_{\emptyset}, S_{i}^{\prime}, S_{-i}\right)$. Therefore, by construction of $S_{-i}$,

(i) $x^{G}\left(h_{\emptyset}, S_{i}, S_{-i}\right)=x^{G}\left(h, S_{i}, C_{-i}\right)$, and

(ii) $x^{G}\left(h_{\emptyset}, S_{i}^{\prime}, S_{-i}\right)=x^{G}\left(h^{\prime}, C_{i}^{\prime}, C_{-i}^{\prime}\right)$,

so $S_{i}$ is obviously $\mathcal{C}$-dominant. Since $i \in N$ was arbitrary, $\left(\mathbb{S}_{i}\left(\theta_{i}\right)\right) \in \operatorname{OSP}^{\mathcal{C}}(G, \theta)$. Since $\theta \in \Theta$ was arbitrary, thus for each $\theta \in \Theta,\left(\mathbb{S}_{i}\left(\theta_{i}\right)\right) \in \operatorname{OSP}^{\mathcal{C}}(G, \theta)$. Since for each $\theta \in \Theta$, $x^{G}\left(h_{\emptyset},\left(\mathbb{S}_{i}\left(\theta_{i}\right)\right)\right)=f(\theta)$, thus $G \mathbf{O S P}^{\mathcal{C}}$-implements $f$ through $\mathbb{S}$, as desired.

\section{References}

Alós-Ferrer, C., Kern, J., And Ritzberger, K. (2011). "Comment on 'Trees and extensive forms."' Journal of Economic Theory 146, 2165-2168.

Alós-Ferrer, C. And Ritzberger, K. (2005a). "Some remarks on pseudotrees." Order 22, 1-9.

Alós-Ferrer, C. And Ritzberger, K. (2005b). "Trees and decisions." Economic Theory 25, 763-798.

Alós-Ferrer, C. And Ritzberger, K. (2008). "Trees and extensive forms." Journal of Economic Theory 143, 216-250. 
Alós-Ferrer, C. And Ritzberger, K. (2016). The Theory of Extensive Form Games. Berlin, Germany: Springer-Verlag Berlin Heidelberg.

Arribillaga, R., Massó, J., And Neme, A. (2017). "Not All Majority-based Social Choice Functions Are Obviously Strategy-proof." Working paper.

Ashlagi, I. And Gonczarowski, Y. (2016). "Stable matching mechanisms are not obviously strategy-proof." Working paper.

Aumann, R. (1964). "Mixed and Behavior Strategies in Infinite Extensive Games." In: Advances in Game Theory, Annals of Mathematics Studies 52. Editors: Dresher, M., Shapley, L., and Tucker, A. Princeton, New Jersey: Princeton University Press.

Aumann, R. (1987). "Correlated Equilibrium as an Expression of Bayesian Rationality." Econometrica 55, 1-18.

Bade, S. and Gonczarowski, Y. (2017). "Gibbard-Satterthwaite Success Stories and Obvious Strategy-proofness." Working paper.

Carroll, G. (2017). "On Mechanisms Eliciting Ordinal Preferences." Forthcoming, Theoretical Economics.

Frank, A. (1947). Het Achterhuis [The Diary, in Dutch]. Amsterdam, the Netherlands: Contact Publishing. Translation: Frank, A. and Massotty, S. (1995). The Diary of a Young Girl: The Definitive Edition. Editors: Frank, O. and Pressler, M. New York, New York: Doubleday.

Gale, D. And Shapley, L. (1962). "College Admissions and the Stability of Marriage." The American Mathematical Monthly 69, 9-15.

GibBard, A. (1973). "Manipulation of Voting Schemes: A General Result." Econometrica $41,587-601$.

Halmos, P. (1950). Measure Theory. New York, New York: Van Nostrand.

HARSANYi, J. (1967). "Games with incomplete information played by 'Bayesian' players, I-III: Part I, The Basic Model." Management Science 14, 159-182.

Isbell, J. (1957). "Finitary games." In: Contributions to the Theory of Games, Volume III, Annals of Mathematics Studies 39. Editors: Dresher, M., Tucker, A., and Wolfe, P. Princeton, New Jersey: Princeton University Press.

JECH, T. (1971). "Trees." The Journal of Symbolic Logic 36, 1-14.

Koppelberg, S. And Monk, J. (1991). "Pseudo-trees and Boolean algebras." Order 8, 359-374.

Kunn, H. (1953). "Extensive games and the problem of information." In: Contributions to the Theory of Games, Volume II, Annals of Mathematics Studies 28. Editors: Kuhn, H. and Tucker, A. Princeton, New Jersey: Princeton University Press.

Li, S. (2016). "Obviously Strategy-Proof Mechanisms." American Economic Review 107, 3257-3287. 
Mackey, G. (1957). "Borel structure in groups and their duals." Transactions of the American Mathematical Society 85, 134-165.

Milgrom, P. And Weber, R. (1982). "A Theory of Auctions and Competitive Bidding." Econometrica 50, 1089-1122.

Myerson, R. (1981). "Optimal Auction Design." Mathematics of Operation Research $6,58-73$.

Osborne, M. And Rubinstein, A. (1994). A Course in Game Theory. Cambridge, Massachusetts: The MIT Press.

Piccione, M. And Rubinstein, A. (1997). "On the Interpretation of Decision Problems with Imperfect Recall." Games and Economic Behavior 20, 3-24.

Pycia, M. And Troyan, P. (2017). "Obvious Dominance and Random Priority." Working paper.

Shapley, L. AND Scarf, H. (1974). "On cores and indivisibility." Journal of Mathematical Economics 1, 23-37.

Troyan, P. (2016). "Obviously Strategy-Proof Implementation of Top Trading Cycles." Working paper.

Vickrey, W. (1961). "Counterspeculation, Auctions, and Competitive Sealed Tenders." The Journal of Finance 16, 8-37. 